Nuclear Physics A456 (1986) 669-700

North-Holland, Amsterdam

\title{
NN TENSOR AND LS POTENTIALS IN A QUARK MODEL WITH QUARK-ANTIQUARK EXCITATIONS
}

\author{
Y. FUJIWARA and K.T. HECHT \\ Physics Department, University of Michigan, Ann Arbor, MI 48109, USA
}

Received 24 January 1986

\begin{abstract}
A more detailed study is made of the NN interaction in a quark model in which the ( $\mathrm{q} \overline{\mathrm{q}}$ ) excitations inherent in the quark-gluon interaction are explicitly incorporated into the model space.

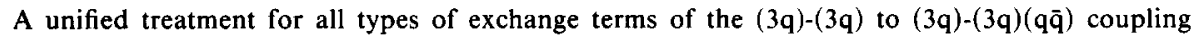
kernels is used to calculate the space parts of the full coupling kernels and their Wigner transforms in complete analytic form. The present investigation focuses on the noncentral parts of the NN interaction. The tensor force gains almost its full strength from coupling kernels of $N \pi$ and $N \rho$ type. If the $\mathrm{N} \pi$ contribution is adjusted to fit the experimental pion-nucleon coupling constant the predicted strength of the full tensor force is in reasonable agreement with that of conventional OBEP's over the range in which the tensor force can act. The $L S$ force gains contributions from both the pure (3q)-(3q) and the coupling kernels, but the dominant contributions (about $60-65 \%$ of the triplet-odd $L S$ potential in the $0.7-1.0 \mathrm{fm}$ range), come from the coupling kernels and particularly from the $\mathrm{N} \omega$ and $\mathrm{N} \rho$ components. The triplet-odd $L S$ potential derived from the full quark-exchange kernel is in remarkably good agreement with the OBEP $L S$ potential over the significant range. Both the tensor and $L S$ potentials are approximated surprisingly well over their full range by the simple ( $q \bar{q})$ exchange terms of our model. The ${ }^{3} \mathrm{P}$ RGM phase shifts are calculated to show that both tensor and $L S$ forces of our quark model are in good agreement with the experimental facts.
\end{abstract}

\section{Introduction}

Since quantum chromodynamics (QCD) is now believed to be the dynamics underlying the strong interaction there have been many attempts to gain a deeper understanding of the NN interaction in terms of QCD-inspired quark models. The most detailed studies of the NN system have been carried out within the framework of the resonating group method [for the many earlier references, see ref. ${ }^{1}$ )], through models in which a gluon exchange potential, usually in a one-gluon exchange approximation through the color analog of the Fermi-Breit interaction, is augmented by a phenomenological confining potential. Despite the successes of such quark potential models, or similar string-based or flux tube potential models ${ }^{2}$ ), these models may not be easy to justify when used for the prediction of single baryon properties which depend on the exact nature and strength of the confining potential (such as the absolute values of baryon masses). Since NN scattering in the low

* Supported by the US National Science Foundation. 
energy regime passes the crucial test of being almost completely insensitive to large changes in the strength of the confinement potential the study of the NN interaction is free of such difficulties.

In the simplest quark models the nucleon is assumed to be a pure three-quark (3q) system. Since the studies of the NN interaction based on the simple (3q)-(3q) models can elucidate only the characteristics of the extreme short range part of this interaction, a whole set of newer quark models have been created in which the quark degrees of freedom of the nucleon are coupled to specific meson fields or exterior scalar fields which confine the quarks to the nucleon interior ${ }^{\star}$.

In an alternative approach $\left.{ }^{1}\right)$ the quark-antiquark $(q \bar{q})$ excitations inherent in the quark-gluon interaction lagrangian have been explicitly incorporated into a quark model of the nucleon in order to study the effects of such excitations on the NN interaction. By staying within the framework of a pure quark model it was the aim of ref. ${ }^{1}$ ) to treat the nucleon and the meson fields on a more equal footing. Quark exchange kernels for the two-nucleon system, in which the (3q) components of the single nucleon wave functions were augmented by $(3 q)(q \bar{q})$ components, led to effective potentials ${ }^{1}$ ) with a medium range attractive part and a greatly reduced repulsive core. This repulsive core shows the strong energy dependence and has numerical values very similar to those of the short range phenomenological terms of the Paris potential.

It was also possible to make good contact with the conventional meson exchange potentials by isolating from the many exchange terms those terms which correspond to the simple exchange of a ( $\mathrm{q} \overline{\mathrm{q}})$ pair between the two nucleons ${ }^{3}$ ). These simple $(\mathrm{q} \overline{\mathrm{q}})$ exchange terms arise only through contributions to the exchange kernels for which the (3q) components carry the quantum numbers of a real nucleon and the $(q \bar{q})$ components have the color singlet character of a real pseudoscalar or vector meson. The effective potentials resulting from these simple $(\mathrm{q} \overline{\mathrm{q}})$ exchanges are in remarkably good agreement with the corresponding OBEP's for $R \geqslant 1.2 \mathrm{fm}$ and have the same qualitative radial features over an even wider range. Moreover, they have all the characteristics of conventional OBEP's in their dependence on nucleon $\left(\sigma_{1} \cdot \sigma_{2}\right)$ and $\left(\tau_{1} \cdot \tau_{2}\right)$ factors, and the relative importance and signs of spin-spin, spin-independent central, spin-orbit, and tensor terms $\left.{ }^{3}\right)$. One of the quantitative failures of these simple $(q \bar{q})$ exchange potentials involves the pion tensor term which is too weak by a factor of $\sim 3$, in agreement with the predicted pion-nucleon coupling constant ${ }^{1}$ ) which is also too small by a factor of $\sim 3$. Since a simple $(q \bar{q})$ pair with the quantum numbers of a pion was not expected to give a realistic picture of the pseudoscalar meson with the mass of a real pion a quantitative fit of the OPEP (including its long-range Yukawa tail) was not expected. However, since the quark model of refs. ${ }^{1,3}$ ) can pinpoint such specific pieces of the NN interaction in terms of the corresponding pieces of the quark exchange kernels, it is one of the advantages

* For many references to the little bag models, cloudy bag models, soliton and skyrmion models, the Friedberg-Lee and other non-linear $\sigma$ models, see e.g. refs. ${ }^{26-31}$ ) of ref. ${ }^{1}$ ). 
of this model that such terms can be isolated and improved to give a more realistic picture of the $\mathrm{NN}$ interaction.

Although the full central potentials gain a medium range attractive part through the ( $\mathrm{q} \overline{\mathrm{q}}$ ) excitations incorporated into the quark model of refs. ${ }^{1,3}$ ), this attractive part was too weak to bind the deuteron or fit the low energy phase shifts. Recently, however, it has been shown ${ }^{4}$ ) that additional central terms are a natural part of such a model. These arise through $(\mathrm{q} \overline{\mathrm{q}})(\mathrm{q} \overline{\mathrm{q}})$ excitations with the quantum numbers of a scalar $(\sigma$ or $\delta)$ meson which are generated by RPA-type off-shell terms and which, like the $(q \bar{q})$ pair creation terms of refs. $\left.{ }^{1,3}\right)$, are a natural part of the full Fermi-Breit quark-gluon interaction. In ref. ${ }^{4}$ ) it is shown that the inclusion of such terms leads to quark exchange kernels which give a semiquantitative fit of the experimental low energy NN scattering data.

Ref. ${ }^{1}$ ) focused on the nature of the model and the single nucleon properties used to fit the four model parameters. Improved single nucleon wave functions were

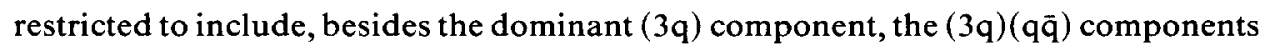
with the 24 possible spin, isospin and color combinations for the energetically lowest p-wave relative motion function. Ref. ${ }^{3}$ ) examined the contributions to the kernels arising from terms coupling the small $(3 q)-(3 q)(q \bar{q})$ components to the dominant (3q)-(3q) components of the NN system in order to isolate those terms of the full exchange kernels which correspond to the exchange of a simple $(\mathrm{q} \overline{\mathrm{q}})$ pair between the two nucleons. These were evaluated in explicit analytic form and their Wigner transforms led to the simple $(q \bar{q})$ exchange potentials which could be compared with conventional meson exchange potentials. Since the simple ( $q \bar{q})$ exchange potentials involve only two of the 25 possible exchange terms of the $(3 q)-(3 q)(q \bar{q})$ to $(3 q)-(3 q)$ coupling kernels and only six of the 24 possible spin, isospin, color combinations of the $(3 q)(q \bar{q})$ components, ref. $^{3}$ ) gives only a very small selection of the total number of possible coupling kernels. It is the purpose of the present investigation to give the details of the mathematical framework needed to evaluate the full exchange kernels. Since the simple $(\mathrm{q} \overline{\mathrm{q}})$ exchange terms dominate only at long range this is vital for a detailed undertstanding of the full potentials. It will be shown that a unified treatment for all types of exchange terms can be used to calculate the full coupling kernels and, in particular their Wigner transforms, in complete analytic form. This makes it possible to separate all terms of the NN interaction into central, spin-orbit and tensor terms including the contributions of the more complicated exchange terms which dominate at extremely short range. However, the present study focuses on the tensor and spin-orbit terms. There are several reasons: (i) tensor and $L S$ terms are relatively weak compared with the strength of the full interaction. This makes it possible to use the Wigner transforms as a direct measure of the potentials of these peripheral terms of the interaction. The Wigner transforms are ideal to study the relative importance, range, and degrec of nonlocality (sensitivity to energy dependence) of the many contributors to the exchange kernels; (ii) the full central potentials at very short range are the result 
of partial cancellation of strong repulsive and attractive contributions and are therefore subject to greater uncertainty than the tensor and $L S$ terms. At extremely short range the central terms may gain significant contributions from terms which are of second order in the $c_{\alpha}$, the amplitudes of the small ( $\left.3 q\right)(q \bar{q})$ components in the single nucleon wave function. These complicated terms have been neglected up to now; (iii) the central potentials have been shown ${ }^{4}$ ) to gain important contributions through the exchange kernels coupling the $(3 q)-(3 q)(q \bar{q})(q \bar{q})$ components to the dominant $(3 q)-(3 q)$ components of the $N N$ system, where the small $(3 q)(q \bar{q})(q \bar{q})$ components of the single nucleon are generated by RPA-type terms of the FermiBreit quark-gluon interaction, and where the $(\mathrm{q} \overline{\mathrm{q}})(\mathrm{q} \overline{\mathrm{q}})$ pieces are of $\sigma$ or $\delta$ scalar meson type. Some of the $\sigma$ and $\delta$ components of this type have been found ${ }^{4}$ ) to have amplitudes, $c_{\beta}$, of the same order as the $c_{\alpha}$. However, such coupling terms make contributions only to the central potentials. Their full elucidation will be the subject of a future study. Since $L S$ and tensor terms gain contributions only from the pure (3q)-(3q) kernels and the coupling kernels of $(3 q)(q \bar{q})$ type, and since they are not complicated by cancellations of important terms at extreme short range, they can be expected to be the most reliable terms at the present stage of development of our quark model.

Sect. 2 gives the construction of the coupling kernels of $(3 q)(q \bar{q})$ type concentrating on the complex generator form of these kernels, in particular their dependence on exchange and interaction type. The details of the transformations to RGM form and the construction of their Wigner transforms are left to an appendix. Since the range and degree of nonlocality of the effective potentials is determined largely through the exponential factors of the Wigner transforms, these are discussed in some detail in sect. 2. Sect. 3 gives a discussion of the tensor and spin-orbit terms, based on the coupling terms of $(3 q)(q \bar{q})$ type of sect. 2 and the pure (3q)-(3q) Wigner transforms from a previous analysis ${ }^{5}$ ). Contributions to the tensor force from the pure $(3 q)-(3 q)$ sector, from the many hidden color channels, and from (3q)(q) $)$ components with a (3q) piece of $\Delta$ type, are all shown to be essentially negligible. The tensor force almost gains its full strength from the $\mathrm{N} \rho$ and $\mathrm{N} \pi$ coupling kernels. However, the $\mathrm{N} \pi$ contribution must be adjusted to fit the experimental nucleon-pion coupling constant. The $L S$ force gains contributions from both the pure (3q)-(3q) and the coupling kernels but is dominated by the latter. In the $0.7-1.0 \mathrm{fm}$ range, e.g., about $60-65 \%$ of the triplet-odd $L S$ potential results from the coupling kernels. The dominant contributions come from the $\mathrm{N} \omega$ and $\mathrm{N} \rho$ components. Both the tensor and $L S$ potentials are approximated surprisingly well over their full range by the simple $(\mathrm{q} \overline{\mathrm{q}})$ exchange potentials.

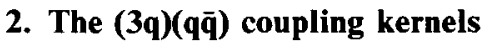

A detailed discussion of the coupling kernels of simple $(\mathrm{q} \overline{\mathrm{q}})$ mesonic exchange type has been given in the earlier ref. ${ }^{3}$ ). Since much of the analysis of ref. $^{3}$ ) is 
applicable without major modification to the evaluation of the full ( $3 q$ )( $q \bar{q})$ coupling kernels, only a few expressions are repeated. In particular, the factoring of the coupling kernels into orbital and spin, isospin, color parts, and subsequent separation into central, $L S$, and tensor terms are effected through the techniques given in detail in ref. ${ }^{3}$ ). However, since only very simple exchange terms were needed in ref. ${ }^{3}$ ), the details for the evaluation of the orbital factors were deferred. By using an expansion of the exchange kernels in terms of double coset generators a unified treatment for all types of exchange terms can be used to calculate the space parts for the full coupling kernels, and these are to be presented here. The new ingredients of this paper therefore are the complete expressions for the full GCM coupling kernels, the transformation formula to the RGM form, and the construction of the Wigner transforms (in appendix A), as well as an analysis of the exchange kernels made possible by the simple analytical expressions for their exponential factors.

The RGM kernels are to be calculated with the improved single nucleon wave functions of refs. $\left.{ }^{1.3}\right)$ in which the $(3 q)$ component is augmented by $(3 q)(q \bar{q})$ components

$$
\phi_{\mathrm{N}}=c_{0} \phi_{0}(3 q)+\sum_{\alpha=1}^{24} c_{\alpha} \phi_{\alpha}((3 q)(q \bar{q})),
$$

where the $\phi_{\alpha}$ are expressed in terms of two-cluster RGM wave functions by

$$
\begin{aligned}
& \phi_{\alpha S M_{S} T M_{T}}((3 \mathrm{q})(\mathrm{q} \overline{\mathrm{q}})) \\
& \quad=i N_{\alpha \alpha}^{-1 / 2} \mathscr{A}\left[\chi_{I=1}^{\text {h.o }}(\boldsymbol{\rho})\left[\phi_{S_{2} T_{2}(\lambda \lambda)}(3 \mathrm{q}) \phi_{S_{3} T_{3}(\lambda \lambda)}(\mathrm{q} \overline{\mathrm{q}})\right]_{\left.S_{23} T(00)\right)}\right]_{S M_{S} T M_{T}} .
\end{aligned}
$$

The 24 possible spin, isospin, color combinations $\left[S_{2} T_{2}(\lambda \lambda) S_{3} T_{3}(\lambda \lambda)\right] S_{23} T(00)$ are enumerated in table 1 of ref. $\left.{ }^{1}\right)$. The square brackets denote angular momentum ( $l$ and $S)$, isospin, and color coupling. As in ref. $\left.{ }^{1}\right)$ the $(3 \mathrm{q})-(\mathrm{q} \overline{\mathrm{q}})$ relative motion function for the energetically lowest $\mathrm{p}$-wave is approximated by a harmonic oscillator function, $\chi^{\text {h.o. }}$.

The improved single-nucleon function of eq. (1) is used to evaluate the exchange kernels for the two-nucleon system

$$
G\left(\boldsymbol{R}, \boldsymbol{R}^{\prime}\right)=c_{0}^{4} G_{0}\left(\boldsymbol{R}, \boldsymbol{R}^{\prime}\right)+c_{0}^{3} \sum_{\alpha=1}^{24} c_{\alpha}\left[G_{\alpha}\left(\boldsymbol{R}, \boldsymbol{R}^{\prime}\right)+G_{\alpha}\left(\boldsymbol{R}^{\prime}, \boldsymbol{R}\right)^{\dagger}\right]+\cdots
$$

As in refs. ${ }^{1,3}$ ) terms of second and higher order in the $c_{\alpha}$ are neglected, and the coupling kernels, $G_{\alpha}$, are further separated into exchange and interaction types

$$
G_{\alpha}\left(\boldsymbol{R}, \boldsymbol{R}^{\prime}\right)=\sum_{P} C_{P} \sum_{i=1}^{6} \sum_{\mathcal{C}=\mathrm{M}, \mathrm{D}} G_{P, i}^{O, \alpha}\left(\boldsymbol{R}, \boldsymbol{R}^{\prime}\right) .
$$

The factoring of these coupling kernels into orbital and spin, isospin, color parts is carried out most efficiently by introducing a vector coherent state of the p-wave oscillator function ${ }^{3}$ ) of eq. (2). This technique also makes it possible to discuss 
separately the central, $L S$, and tensor kernels generated from the coupling kernel of eq. (4). With eqs. (7) and (26) of ref. ${ }^{3}$ ) one can express

$$
G_{P, i}^{O, \alpha}\left(\boldsymbol{R}, \boldsymbol{R}^{\prime}\right)=-\frac{4}{\pi} \alpha_{s} \hbar c N_{\alpha \alpha}^{-1 / 2} \sum_{\Omega=\mathrm{C}, I S, \mathrm{~T}} \mathcal{M}_{P, i}^{O, \Omega}\left(\boldsymbol{R}, \boldsymbol{R}^{\prime}\right) X_{P, i}^{O, \alpha \Omega},
$$

where the spin, isospin, color factors, $X_{P, i}^{O, \alpha \Omega}$ are given through eqs. (27) and (16) of ref. ${ }^{3}$ ). The space parts of these RGM kernels are evaluated through their Bargmann transforms or complex generator coordinate (GCM) kernels ${ }^{6,7}$ ) [see eq. (A.1) of the appendix]. Due to the vector character of the space part of the ( $q \bar{q})$ pair creation interaction, and of the p-wave oscillator function, it becomes useful to express the GCM kernel through cartesian components by

$$
\begin{aligned}
\left(I_{P, i}^{O G C M}\left(z, z^{\prime}\right)\right)_{j k}= & \left(\frac{\partial}{\partial e_{k}}<P A_{\gamma_{1}}\left(\boldsymbol{r}_{01}, z\right) A_{\gamma}(\boldsymbol{\rho}, \boldsymbol{e}) \phi_{0 s}(3 \mathrm{q}) \phi_{0 s}(3 \mathrm{q}) \phi_{0 s}(\mathrm{q} \overline{\mathrm{q}})\right. \\
& \left.\left.\times\left|\delta\left(\boldsymbol{x}_{7}-\boldsymbol{x}_{8}\right) F_{j}^{O}\left(\boldsymbol{x}_{i}, \boldsymbol{x}_{7}\right)\right| A_{y_{0}}\left(\boldsymbol{r}_{00}, z^{\prime}\right) \phi_{0 s}(3 \mathrm{q}) \phi_{0 s}(3 \mathrm{q})\right\rangle\right)_{e=0},
\end{aligned}
$$

from which the space part $\mathcal{M}^{\Omega}\left(\boldsymbol{R}, \boldsymbol{R}^{\prime}\right)$ in (5) is generated for each type $\Omega$ through (A.11) after the Bargmann transformation. In eq. (6), $e$ is a real unit vector with cartesian components $e_{k}$, used in the construction of the p-wave oscillator function. The $\boldsymbol{A}_{\gamma}(\boldsymbol{R}, z)$ are harmonic oscillator coherent states [see eq. (A.2)]. With the 3 dimensional complex Bargmann space variables $z$ and $z^{\prime}$ they are also the kernel functions for the Bargmann transformation. Note that the three $A_{\gamma}$ 's of eq. (6) involve the three types of relative motion vectors, $r_{01}$ from the $\mathrm{cm}$ of the $(3 q)(q \bar{q})$ cluster of one nucleon to the $\mathrm{cm}$ of the $(3 \mathrm{q})$ cluster of the other, $\rho$ from the $\mathrm{cm}$ of the ( $3 q)$ to the $(q \bar{q})$ cluster, and $r_{00}$ from the $\mathrm{cm}$ of one ( $\left.3 q\right)$ cluster to the other. The range parameters with the corresponding reduced mass factors are $\gamma_{1}=$ $\left(15 / 16 b^{2}\right), \gamma=\left(3 / 5 b^{2}\right)$ and $\gamma_{0}=\left(3 / 4 b^{2}\right)$. The spatial vector factor of the (qā) pair creation interaction [see ref. $\left.{ }^{1}\right)$ ], with cartesian components $F_{j}^{\mathscr{O}}\left(\boldsymbol{x}_{i}, \boldsymbol{x}_{7}\right)$, contains the two interaction types $\mathscr{O}=\mathrm{D}$ (derivative) and $\mathscr{O}=\mathbf{M}$ (momentum) type,

$$
\boldsymbol{F}^{\mathrm{D}}\left(\boldsymbol{x}_{i}, \boldsymbol{x}_{7}\right)=\frac{\partial}{\partial \boldsymbol{r}} F(r), \quad \boldsymbol{F}^{\mathrm{M}}\left(\boldsymbol{x}_{i}, \boldsymbol{x}_{7}\right)=\boldsymbol{F}(\boldsymbol{r}) \frac{\partial}{\partial \boldsymbol{x}_{i}},
$$

with

$$
F(r)=\frac{\pi}{2} \frac{\hbar}{m c} \frac{1}{r}=\frac{\pi}{2} x \frac{b}{r}, \quad r=\left|x_{i}-x_{7}\right|, \quad x=\frac{\hbar}{m c} \frac{1}{b} .
$$

As in refs. ${ }^{1,3}$ ) the quarks are laheled with indices $i=1,2,3$ in the single nucleon

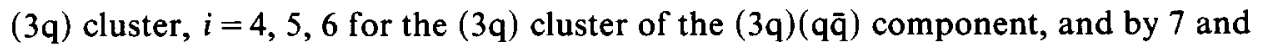
8 for the $q$ and $\bar{q}$ partners of the $(q \bar{q})$ pair. The exchange type is specified by the 
permutation operator, $P$, in the expansion of the full seven-quark antisymmetrizer, and the index $i(i=1, \ldots, 6)$, which characterizes the placement of the potential line in the $(\mathrm{q} \overline{\mathrm{q}})$ pair creation interaction $U_{\mathrm{q} \rightarrow q q \bar{q}}\left(x_{i} ; x_{7}, x_{8}\right)$ [see e.g. figs. $1 \mathrm{~b}$ and 2 of ref. $\left.{ }^{3}\right)$ ]. The $(7 ! / 3 ! 3 ! 1 !)$ permutations, $P$, which exchange quarks between the three clusters with labels $1,2,3 ; 4,5,6$; and 7 can be reduced to eight basic types through an expansion in terms of double coset generators ${ }^{8}$ ) by making use of the antisymmetry of the full (3q) internal wave functions and the antisymmetry of the full two-nucleon (3q)-(3q) wave function of the ket side of the coupling kernel [see eq. (43) of ref. $\left.{ }^{1}\right)$ ]. For the evaluation of the kernels it is useful to characterize the double coset generators by the double coset symbol ${ }^{8}$ ), $d_{a b}$, which gives the sum of the unit matrix elements in the $a b$ th submatrix of the $7 \times 7$ matrix representation of $P$. For the $3+3+1$ three-cluster decomposition of the seven quarks this can be specified by four integers xyuv:

$$
d_{a b}=\left(\begin{array}{ccc}
3-x & u & x-u \\
v & 3-y & y-v \\
x-v & y-u & 1-x-y+u+v
\end{array}\right)
$$

The weighting factors $C_{p}$ of eq. (4) are given by

$$
C_{P}=\delta_{P} \frac{3 ! 3 ! 1 !}{\prod_{a b}\left(d_{a b}\right) !},
$$

where $\delta_{P}= \pm 1$ for even/odd $P$. The eight basic $P$ 's are listed in table 1 together with the index $i$ which, with $P$, specifies the exchange type. Alternately, the exchange type $i(P)$ can be characterized by the two indices $\{\alpha, \beta\}$ which describe the \{final, initial\} cluster position of the quark line for the vertex $i$ of the exchange diagram. Thus $\beta=1$ for $i=1,2,3 ; \beta=2$ for $i=4,5,6$; whereas $\alpha$ is determined by the cluster index of $l$ where $P$ converts the particle-index $l$ into $i$, so that $\alpha=1$ for $l=1,2,3 ; \alpha=2$ for $l=4,5,6$; and $\alpha=3$ for $l=7$. Since $P=e$ and $P=(67)$ terms contribute only to the single nucleon internal energies, there are only six basic $P$ 's which contribute to the two-nucleon exchange kernels. Table 1 shows that these lead to 25 inequivalent types of exchange terms. The GCM exchange kernels are determined completely by the parameters of the interaction functions $\boldsymbol{F}^{\sigma}\left(\boldsymbol{x}_{i}, \boldsymbol{x}_{7}\right)$ and the four integers $x y u v$ and the two indices $\alpha, \beta$, so that quite general expressions can be given. The GCM kernels are easiest to evaluate if the scalar function, $F(r)$, of eq. (7) is expanded in terms of gaussian functions $\exp \left(-\chi^{2} r^{2}\right)$. E.g.

$$
\frac{b}{r}=\frac{2}{\sqrt{\pi}} \int_{0}^{\infty} \mathrm{d}(b \chi) \mathrm{e}^{-\chi^{2} r^{2}} .
$$

Since the derivative operations of eq. (7) commute with the $\chi$-integration, the kernels follow directly from the simple gaussian kernels which have been evaluated 
TABLE 1

The inequivalent exchange types

\begin{tabular}{|c|c|c|c|c|c|c|c|}
\hline No. & $\begin{array}{c}P \\
x y u v\end{array}$ & $i$ & $\alpha \beta$ & No. & $\begin{array}{c}P \\
x y u v\end{array}$ & $i$ & $\alpha \beta$ \\
\hline & e & & & & & & \\
\hline \multirow[t]{2}{*}{$\left.1^{a}\right)$} & 0000 & 4-6 & 22 & 15 & & 1,2 & 11 \\
\hline & & & & 16 & $(37)(36)$ & 3 & 31 \\
\hline $\left.2^{a}\right)$ & (67) & 4,5 & 22 & 17 & 1110 & 4,5 & 22 \\
\hline $\left.3^{a}\right)$ & 0100 & 6 & 32 & 18 & & 6 & 12 \\
\hline 4 & (37) & 1,2 & 11 & 19 & & 1,2 & 11 \\
\hline 5 & 1000 & 3 & 31 & 20 & $(36)(57)$ & 3 & 21 \\
\hline \multirow[t]{2}{*}{6} & & $4-6$ & 22 & 21 & 1211 & 4 & 22 \\
\hline & & & & 22 & & 5 & 32 \\
\hline 7 & & 1,2 & 11 & 23 & & 6 & 12 \\
\hline 8 & (36) & 3 & 21 & & & & \\
\hline 9 & 1111 & 4,5 & 22 & 24 & & 1 & 11 \\
\hline \multirow[t]{2}{*}{10} & & 6 & 12 & 25 & $(36)(27)$ & 2 & 31 \\
\hline & & & & 26 & 2111 & 3 & 21 \\
\hline 11 & & 1,2 & 11 & 27 & & 4,5 & 22 \\
\hline 12 & $(36)(37)$ & 3 & 21 & 28 & & 6 & 12 \\
\hline 13 & 1101 & 4,5 & 22 & & & & \\
\hline 14 & & 6 & 32 & & & & \\
\hline
\end{tabular}

a) Terms 1 (direct) and 2 and 3 contribute only to the internal energy.

by the standard methods $\left.{ }^{6,7,9,10}\right)$. With the gaussian replacement $F(r) \rightarrow u(r)=$ $\exp \left(-\chi^{2} r^{2}\right)$ the GCM kernel of eq. (6) has the general form

$$
\begin{aligned}
\left(I_{P, i}^{O G C M}\left(z, z^{\prime}\right)\right)_{j k}= & {\left[\frac{4 \sqrt{3}}{6+13 \chi^{2} b^{2}}\right]^{3 / 2}\left(-\left[\frac{1}{3 b^{2}}\right]^{1 / 2}\right)\left\{\left(a z^{*}+b z^{\prime}\right)_{j}\left(c z^{*}+\mathrm{d} z^{\prime}\right)_{k}+e \delta_{j k}\right\} } \\
& \times \exp \left[-\frac{1}{2} f^{(0)} z^{* 2}+h^{(0)}\left(z^{*} \cdot z^{\prime}\right)-\frac{1}{2} \frac{\kappa}{6+\kappa}\left(p z^{*}+q z^{\prime}\right)^{2}\right],
\end{aligned}
$$

where

$$
\kappa=\frac{\chi^{2} b^{2}}{1+2 \chi^{2} b^{2}}
$$

and where the parameters $a, b, \ldots$ are given in terms of $\kappa$ and $\kappa$-independent parts $a^{(0)}, b^{(0)}, \ldots$, by

$$
\begin{gathered}
a=a^{(0)}-\frac{\kappa}{6+\kappa} p m, \quad b=b^{(0)}-\frac{\kappa}{6+\kappa} q m, \quad c=c^{(0)}-\frac{\kappa}{6+\kappa} p k, \\
d=d^{(0)}-\frac{\kappa}{6+\kappa} q k, \quad e=e^{(0)}-\frac{\kappa}{6+\kappa} m k .
\end{gathered}
$$


The dependence on exchange type $P, i$ is given through $x y u v$ and $\alpha, \beta$ (see table 1); with

$$
\begin{gathered}
f^{(0)}=\frac{4}{9}(x-u)-\frac{1}{5}, \quad p=2 \sqrt{\frac{1}{5}}\left[2-\frac{7}{3}(x-u)+\varepsilon_{\alpha}\right], \\
h^{(0)}-2 \sqrt{\frac{1}{5}}\left[1-\frac{1}{3}(x+u)\right], \quad q=\varepsilon_{\beta}, \\
k=\sqrt{5}\left[\frac{7}{10}(x-u-2)+\frac{7}{6}(y-v)+\delta_{\alpha}\right], \\
c^{(0)}=\frac{2}{3}(x-u)+\frac{1}{3}(y-v)-\frac{2}{5}, \\
d^{(0)}=\sqrt{\frac{1}{5}}\left[1+\frac{1}{2}(x+u)-\frac{5}{6}(y+v)\right],
\end{gathered}
$$

where the coefficients $c$ and $d$ are the same for both $\mathrm{D}$ and M-type factors of the interaction, whereas $a, b$, and $e$ must be given separately.

For D-type:

$$
a^{(0)}=b^{(0)}=e^{(0)}=0, \quad m=-6 .
$$

For M-type:

$$
\begin{gathered}
a^{(0)}=\sqrt{\frac{1}{5}}\left[\frac{1}{3}(x-u)+\varepsilon_{\alpha}\right], \quad b^{(0)}=-\frac{1}{2} \varepsilon_{\beta}, \\
e^{(0)}=\frac{1}{2} \sqrt{5}\left[\delta_{\alpha}-\frac{1}{10}(x-u-2)-\frac{1}{6}(y-v)\right], \quad m=\frac{5}{2} .
\end{gathered}
$$

The $\alpha, \beta$-dependent parameters are given by

$$
\varepsilon_{1}=1, \quad \varepsilon_{2}=\varepsilon_{3}=-1 ; \quad \delta_{1}=0, \quad \delta_{2}=-\frac{2}{5}, \quad \delta_{3}=\frac{3}{5} .
$$

Since the sign of the coefficient of the $\left(z^{*} \cdot z^{\prime}\right)$ term in the exponential plays a special role, we note that this coefficient, viz,

$$
\frac{2}{\sqrt{5}}\left[1-\frac{1}{3}(x+u)-\frac{\chi^{2} b^{2}}{6+13 \chi^{2} b^{2}}\left(2-\frac{7}{3}(x-u)+\varepsilon_{\alpha}\right) \varepsilon_{\beta}\right],
$$

is negative for only two of the possible exchange types, exchange types 24 and 27 of table 1, with $x u=21$, and $\alpha \beta=11$ and 22, respectively.

The GCM kernels of eq. (11) are converted into standard RGM kernels in completely analytic form with the use of the formulas given in appendix $\mathrm{A}$, where their Wigner transforms are also constructed. Since the $(3 q)(q \bar{q})$ admixtures in the single-nucleon wave functions appear symmetrically in both bra and ket, the full

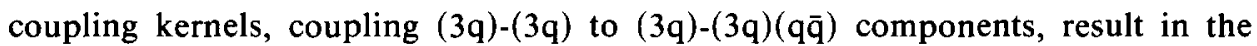
symmetrized form, as in eq. (3). With this effect taken into account, the full Wigner transform of the $(3 q)(q \bar{q})$ coupling kernel is expressed as

$$
\begin{aligned}
G_{\mathrm{W}}(\boldsymbol{R}, \boldsymbol{P})= & G_{\mathrm{W}}^{\mathrm{C}}(\boldsymbol{R}, \boldsymbol{P})+G_{\mathrm{W}}^{L S}(\boldsymbol{R}, \boldsymbol{P})(\boldsymbol{L} \cdot \boldsymbol{S})+G_{\mathrm{W}}^{\mathrm{T}}(\boldsymbol{R}, \boldsymbol{P}) S_{12}(\hat{\boldsymbol{R}}, \hat{\boldsymbol{R}}) \\
& +G_{\mathrm{W}}^{\mathrm{T}}(\boldsymbol{R}, \boldsymbol{P}) S_{12}(\hat{\boldsymbol{P}}, \hat{\boldsymbol{P}})+G_{\mathrm{W}}^{\mathrm{T} \prime \prime}(\boldsymbol{R}, \boldsymbol{P}) S_{12}(\hat{\boldsymbol{R}}, \hat{\boldsymbol{P}})
\end{aligned}
$$

where $\hat{\boldsymbol{R}}$ and $\hat{\boldsymbol{P}}$ are unit vectors and $S_{12}(\boldsymbol{U}, \boldsymbol{V})$ is defined by

$$
S_{12}(\boldsymbol{U}, \boldsymbol{V})=\frac{3}{2}\left(\sigma_{1} \cdot U\right)\left(\sigma_{2} \cdot V\right)+\frac{3}{2}\left(\sigma_{2} \cdot U\right)\left(\sigma_{1} \cdot V\right)-(U \cdot V)\left(\sigma_{1} \cdot \sigma_{2}\right)
$$


The central, $L S$, and tensor Wigner transforms in (12) are given by

$$
\begin{gathered}
G_{\mathrm{W}}^{\Omega l}(\boldsymbol{R}, \boldsymbol{P})=c_{0}^{3} \sum_{\alpha=1}^{24} c_{\alpha} G_{\mathrm{W}}^{\alpha \Omega 2}(\boldsymbol{R}, \boldsymbol{P}), \\
G_{\mathrm{W}}^{\alpha \Omega}(\boldsymbol{R}, \boldsymbol{P})=\sum_{\boldsymbol{P}} C_{P} \sum_{i=1}^{6} \sum_{\sigma=\mathrm{D}, \mathrm{M}} G_{P, i \mathrm{~W}}^{O, \alpha \Omega}(\boldsymbol{R}, \boldsymbol{P}), \\
G_{P, i \mathrm{~W}}^{O, \alpha \Omega}(\boldsymbol{R}, \boldsymbol{P})=\alpha_{s} x^{2} m c^{2} N_{\alpha \alpha}^{-1 / 2} \frac{4}{\sqrt{3 \pi}} f_{P, i} X_{P, i}^{O, \alpha \Omega} \\
\times \int_{0}^{\infty} \mathrm{d}(b \chi)\left[\frac{4 \sqrt{3}}{6+13 b^{2} \chi^{2}}\right]^{3 / 2} g_{\mathrm{w}}^{\Omega}\left(\boldsymbol{R}, \boldsymbol{P} ; \chi^{2}\right) .
\end{gathered}
$$

The phase factor $f_{P, i}$ is given by

$$
f_{P, i}=f_{x y u v, \alpha \beta}= \begin{cases}(-1)^{S+T+1} & \text { for } x u=21 ; \alpha \beta=11 \text { or } 22 \\ +1 & \text { otherwise } .\end{cases}
$$

The $g_{\mathrm{W}}^{\Omega}$ are functions of $\mathcal{O}$ and exchange type, $P, i$, through the coefficients $a, \ldots, h$. They are given by

$$
\begin{aligned}
& g_{\mathrm{w}}^{\Omega}\left(\boldsymbol{R}, \boldsymbol{P} ; \chi^{2}\right)=\left(\frac{4}{D_{\mathrm{w}}}\right)^{3 / 2} \exp \left[-\eta\left(\frac{\boldsymbol{R}}{b}\right)^{2}-\zeta\left(\frac{b \boldsymbol{P}}{\hbar}\right)^{2}\right] \cos \left(\xi \frac{\boldsymbol{R} \cdot \boldsymbol{P}}{\hbar}\right) \\
& \times\left\{\begin{array} { l l } 
{ v ^ { ( 0 ) } + v ^ { ( 2 ) } ( \frac { R } { b } ) ^ { 2 } + u ^ { ( c ) } ( \frac { b P } { \hbar } ) ^ { 2 } + u ^ { ( s ) } ( \frac { R \cdot P } { \hbar } ) \operatorname { t a n } ( \xi \frac { R \cdot P } { \hbar } ) } \\
{ v ^ { L S } } \\
{ v ^ { ( 2 ) } ( \frac { R } { b } ) ^ { 2 } ; \quad u ^ { ( c ) } ( \frac { b P } { \hbar } ) ^ { 2 } ; \quad u ^ { ( s ) } \frac { | R | | P | } { \hbar } }
\end{array} \quad \text { for } \left\{\begin{array}{l}
\Omega=\mathrm{C} \\
\Omega=L S \\
\Omega=\mathrm{T} ; \mathrm{T}^{\prime} ; \mathrm{T}^{\prime \prime} .
\end{array}\right.\right.
\end{aligned}
$$

Since the explicit expressions for the coefficients $D_{\mathrm{w}}, \eta, \zeta, \xi, v^{(0)}, v^{(2)}, u^{(c)}, u^{(s)}$ and $v^{L S}$ are somewhat complicated when given as general functions of the parameters of the GCM kernel of eq. (11), it will be more useful to tabulate results, obtained through the techniques of appendix $\mathrm{A}$, for the various exchange and interaction types. Since the $\kappa$-dependence of the coefficients is given in completely analytic form, the $\chi$ integral of eq. (10) can be carried out, and final results can be given in terms of some special functions related to standard error functions. The coefficients $D_{\mathrm{w}}, \eta, \zeta, \xi$, which specify the common exponential factor of $g_{\mathrm{w}}^{\Omega}$ are given in table 2 . They depend on the exchange type only through the parameters $x u$ and $\alpha \beta$. (Since $\varepsilon_{\alpha=2}=\varepsilon_{\alpha=3}$, see eq. (11), note that these coefficients are the same for exchange types with $\alpha \beta=2 \beta$ and $3 \beta$.)

The exponential factors can be put in the form $\exp \left[-\left(\boldsymbol{R} / R_{0}\right)^{2}-\left(E / E_{0}\right)\right]$, with $R_{0}=b / \sqrt{\eta}$ and $E_{0}=\hbar^{2} / M_{\mathrm{N}} b^{2} \zeta$. Although the range and sensitivity to energy of the interaction are governed by both the polynomial and exponential factors of $g_{\mathrm{w}}^{\Omega}$, the 
TABLE 2

Coefficients for the exponential factors of gaussian $g_{\mathrm{W}}$

\begin{tabular}{ccccc}
\hline$\left.x u ; \alpha \beta^{\mathrm{a}}\right)$ & $\left(4 / \sqrt{5} D_{\mathrm{W}}\right)$ & $\eta$ & $\zeta$ & $\xi$ \\
\hline $00 ; 11$ & $\frac{5(6+\kappa)}{4(15-4 \kappa)}$ & $\frac{15 \kappa}{15-4 \kappa}$ & $\frac{3-4 \kappa}{3(15-4 \kappa)}$ & $\frac{8 \kappa}{15-4 \kappa}$ \\
$00 ; 22$ & $\frac{6+\kappa}{4(3+\kappa)}$ & 0 & $\frac{3-4 \kappa}{15(3+\kappa)}$ & 0 \\
$10 ; 11$ & $\frac{15(6+\kappa)}{130+3 \kappa}$ & $\frac{15(5+3 \kappa)}{130+3 \kappa}$ & $\frac{12}{130+3 \kappa}$ & $\frac{2(20-3 \kappa)}{130+3 \kappa}$ \\
$10 ; 21$ & $\frac{15(6+\kappa)}{26(5+2 \kappa)}$ & $\frac{15}{26}$ & $\frac{2(3-4 \kappa)}{13(5+2 \kappa)}$ & $\frac{4}{13}$ \\
$10 ; 22$ & $\frac{15(6+\kappa)}{2(65-6 \kappa)}$ & $\frac{5(15+14 \kappa)}{2(65-6 \kappa)}$ & $\frac{2(27-4 \kappa)}{9(65-6 \kappa)}$ & $\frac{4(15+4 \kappa)}{3(65-6 \kappa)}$ \\
$11 ; 11$ & $\frac{15(6+\kappa)}{84-37 \kappa}$ & $\frac{5(12+29 \kappa)}{84-37 \kappa}$ & $\frac{4(75-64 \kappa)}{9(84-37 \kappa)}$ & $-\frac{2(12-151 \kappa)}{3(84-37 \kappa)}$ \\
$11 ; 21$ & $\frac{15(6+\kappa)}{4(21-\kappa)}$ & $\frac{5(3+2 \kappa)}{21-\kappa}$ & $\frac{75-4 \kappa}{9(21-\kappa)}$ & $-\frac{2(3+2 \kappa)}{3(21-\kappa)}$ \\
$11 ; 12$ & $\frac{15(6+\kappa)}{4(21+20 \kappa)}$ & $\frac{5(3+5 \kappa)}{21+20 \kappa}$ & $\frac{25(3-4 \kappa)}{9(21+20 \kappa)}$ & $-\frac{2(3-40 \kappa)}{3(21+20 \kappa)}$ \\
$11 ; 22$ & $\frac{15(6+\kappa)}{7(12+5 \kappa)}$ & $\frac{5}{7}$ & $\frac{4(75-16 \kappa)}{63(12+5 \kappa)}$ & $-\frac{2}{21}$ \\
$21 ;\left(\begin{array}{l}11 \\
12\end{array}\right)$ & $\frac{15(6+\kappa)}{4(13+4 \kappa)}$ & $\frac{5(21+8 \kappa)}{4(13+4 \kappa)}$ & $\frac{51-4 \kappa}{9(13+4 \kappa)}$ & $\frac{27-4 \kappa}{3(13+4 \kappa)}$ \\
$21 ;\left(\begin{array}{l}21 \\
22\end{array}\right)$ & $\frac{15(6+\kappa)}{52+27 \kappa}$ & $\frac{5(21+11 \kappa)}{52+27 \kappa}$ & $\frac{4(51-16 \kappa)}{9(52+27 \kappa)}$ & $\frac{2(54+19 \kappa)}{3(52+27 \kappa)}$ \\
\hline
\end{tabular}

a) Terms with $\alpha \beta=2 \beta$ and $3 \beta$ have identical exponential factors.

parameters $R_{0}$ and $E_{0}$ are among the most sensitive indicators of the range and degree of nonlocality of the various terms of the interaction. The numerical values of $R_{0}$ and $E_{0}$ are shown in table 3 , using the value $b=0.5235 \mathrm{fm}$ from the parameter set of ref. ${ }^{1}$ ).

The values of $R_{0}$ and $E_{0}$ are shown for two values of $\kappa ; \kappa=0$ and $\kappa=\frac{1}{2}$, corresponding to $F(r)=1$ and $F(r)=\delta(r)$; that is an extreme long and short range limit in the $r$-dependence of the ( $(\mathrm{q} \overline{\mathrm{q}})$ pair creation interaction. Since the dominant term in $F(r)$ is $1 / r$ the actual values of $R_{0}$ and $E_{0}$ should lie between these two extremes. Table 3 shows that the longest range terms in the interaction with the smallest degree of nonlocality are of exchange type $P=(37)$ with $i=1,2(\alpha \beta=11)$ or $i=3(\alpha \beta=31)$, see also table 1 . These exchange terms were identified as the simple color singlet ( $\mathrm{q} \overline{\mathrm{q}}$ ) exchange terms in ref. $^{3}$ ), where it was also shown that they dominate the long range part of the interaction. Table 3 shows that exchange terms with $P=(36)(37)$ and $i=1,2$, or 3 have values of $R_{0}$ and $E_{0}$ identical with those for $P=(37) i=1,2$, or 3 . The detailed calculations of ref. $\left.{ }^{3}\right)$, however, show 
TABLE 3

Range and energy parameters for the exponential factors

\begin{tabular}{|c|c|c|c|c|c|}
\hline & \multirow{2}{*}{$x u ; \alpha \beta$} & \multicolumn{2}{|c|}{$R_{0}(\mathrm{fm})$} & \multicolumn{2}{|c|}{$E_{0}(\mathrm{MeV})$} \\
\hline & & $\kappa=0$ & $\kappa=\frac{1}{2}$ & $\kappa=0$ & $\kappa=\frac{1}{2}$ \\
\hline $\begin{array}{l}P=(37) \\
(36)(37)\end{array}$ & $\begin{array}{l}10 ; 11 \\
10 ; 21 \\
10 ; 22\end{array}$ & 0.689 & $\begin{array}{l}0.608 \\
0.689 \\
0.556\end{array}$ & 1639 & $\begin{array}{l}1658 \\
5902 \\
1689\end{array}$ \\
\hline $\begin{array}{l}P=(36) \\
(37)(36) \\
(36)(57)\end{array}$ & $\begin{array}{l}11 ; 11 \\
11 ; 21 \\
11 ; 12 \\
11 ; 22\end{array}$ & 0.619 & $\begin{array}{l}0.368 \\
0.530 \\
0.556 \\
0.619\end{array}$ & 381 & $\begin{array}{r}519 \\
382 \\
1689 \\
516\end{array}$ \\
\hline$P=(36)(27)$ & $21 ; \begin{array}{l}11 \\
12 \\
21 ; \begin{array}{l}21 \\
22\end{array}\end{array}$ & 0.368 & $\begin{array}{l}0.363 \\
0.368\end{array}$ & 347 & $\begin{array}{l}417 \\
519\end{array}$ \\
\hline
\end{tabular}

that the simple color singlet ( $\mathrm{q} \overline{\mathrm{q}}$ ) exchange terms dominate for $R \geqslant 1.2 \mathrm{fm}$. It is, of course, not surprising that the polynomial factors of the kernels should play a prominent role in the region where $R$ is appreciably greater than $R_{0}$.

Exchange terms of type $P=(36)(37), i=1,2$, or 3 do make the next most important contributions in the $R=1.0-1.2 \mathrm{fm}$ region. It is interesting to note that these exchange terms also involve the exchange of a color singlet $(\mathrm{q} \overline{\mathrm{q}})$ pair between the two nucleons although they are of the more complicated three cluster variety [see fig. $2 \mathrm{~b}$ of ref. ${ }^{3}$ )]. The most complicated cxchange terms of the threc cluster varicty arc those with $\boldsymbol{P}=(36)(27)$. Table 3 shows that these terms must be expected to be of extremely short range and have the largest degree of nonlocality. The rather small values of $E_{0}$ indicates the fairly strong energy dependence of these terms. The detailed calculations show that these are among the dominant contributors to the central terms of the NN interaction at $\boldsymbol{R}=0$ and $\boldsymbol{P}=0$.

It is also instructive to compare the range and energy parameters $R_{0}$ and $E_{0}$ of table 3 with those of the (3q)-(3q) exchange kernel, $G_{0}$, of eq. (3) and also with those of the $(3 q)-(3 q)(q \bar{q})^{2}$ coupling kernels introduced in ref. $\left.{ }^{4}\right)$. The longest range parameter for the (3q)-(3q) piece is given by the knock-on exchange term of the color-magnetic contact interaction. With $b=0.5235 \mathrm{fm}$, one finds $R_{0}=0.60 \mathrm{fm}$ for this term (the same value as that for the (3q)-(3q) norm kernel), and $E_{0}=\infty$. This apparently explains why this $P_{36}\left(\lambda_{3} \cdot \lambda_{6}\right)\left(\boldsymbol{\sigma}_{3} \cdot \sigma_{6}\right)$ exchange term is the most important one in the (3q)-(3q) exchange kernel. For the $(3 q)-(3 q)(q \bar{q})^{2}$ coupling kernels, the longest range parameter is given by the single quark exchange term of simple (q $\bar{q})^{2}$ exchange type; with $R_{0}=0.61-0.69 \mathrm{fm}, E_{0}=2032-1166 \mathrm{MeV}$, depending on the explicit $r$-dependence of the RPA-type off-shell interaction. As expected, the $(\mathrm{q} \overline{\mathrm{q}})$ and $(\mathrm{q} \overline{\mathrm{q}})^{2}$ excitations of the single-nucleon wave function create new kinds of 
interactions, including in particular long range terms which overwhelm the simple $(3 q)-(3 q)$ interaction in the nucleon surface region.

The $\kappa$-dependence of the coefficients $v^{(0)}, v^{(2)}, v^{L S}, u^{(c)}$, and $u^{(s)}$ of the Wigner transforms for the gaussian kernels is given through tables 4-6 of appendix B. With these tabulations full analytic expressions for the Wigner transforms can be constructed. These are ideal for a study of the relative importance of different exchange terms and different $(3 q)(q \bar{q})$ components of the single-nucleon wave functions. They also make it possible to construct full equivalent local potentials. For the weaker long range components of the $\mathrm{NN}$ interaction the Wigner transforms may by themselves be good approximations for the corresponding effective potentials.

\section{Tensor and $L S$ potentials}

Since the tensor and spin-orbit terms are relatively weak compared with some of the most prominent short range central terms of the $\mathrm{NN}$ interaction, the Wigner transforms by themselves can be taken to give a direct measure of these peripheral terms of the NN interaction. It will be shown that coupling kernels of $(3 q)(q \bar{q})$ type are the most important contributors to the tensor and $L S$ potentials. Since the coupling kernels are to very good approximation given by terms linear in the single-nucleon $c_{\alpha}$ it becomes possible to study the relative importance of individual $c_{0}^{3} c_{\alpha} G_{\mathrm{W}}^{\alpha \Omega}(\boldsymbol{R}, \boldsymbol{P})$ contributors to the effective potentials by turning on one term of the Wigner transform at a time. Wigner transforms for the pure $(3 q)-(3 q)$ parts of the kernel have been given previously ${ }^{5}$ ).

Since our main interest is in low-energy NN scattering, the Wigner transforms with $\boldsymbol{P}=\mathbf{0}$ are used to analyse the relative importance of the various coupling term contributions and the (3q)-(3q) contribution coming from the noncentral pieces of the Breit interaction. The model parameters used in refs. ${ }^{1,3}$ ) were carefully selected in ref. ${ }^{1}$ ) to be consistent with important single-nucleon properties. Since the introduction of the $(\mathrm{q} \overline{\mathrm{q}})^{2}$ excitations in ref. $\left.{ }^{4}\right)$ reduces the magnitudes of the $c_{\alpha}$ for the $(3 q)(\mathrm{q} \bar{q})$ components by small amounts, the new set of $c_{\alpha}$ from ref. $\left.{ }^{4}\right)$ is used for the numerical calculations in this paper. However, these lead only to very small changes, since the new $c_{\alpha}$ and $c_{0}$ are almost identical with those quoted in table 1 of ref. ${ }^{\prime}$ ) [see ref. $^{4}$ )].

Figs. 1 through 5 show the various contributors to the tensor force. The contribution of the pure (3q)-(3q) part of the kernel, $c_{0}^{4} G_{0 \mathrm{w}}^{T}(\boldsymbol{R}, 0)$, with maximum values of less than $4 \mathrm{MeV}$, is shown to be negligible in fig. 1a. This agrees with the analysis of the simple (3q)-(3q) model $^{12}$ ) where the inclusion of the tensor force in the Breit quark interaction has been shown to lead to essentially no changes in the predicted phase shifts. Fig. 1b shows the contributions to the tensor force from the many hidden

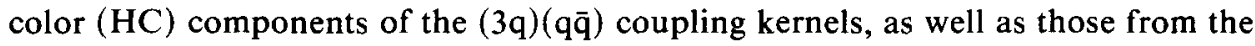
$(3 q)(q \bar{q})$ components where the (3q) piece carries the quantum number of a $\Delta$. Both are again shown to make negligible contributions to the tensor force, despite the 

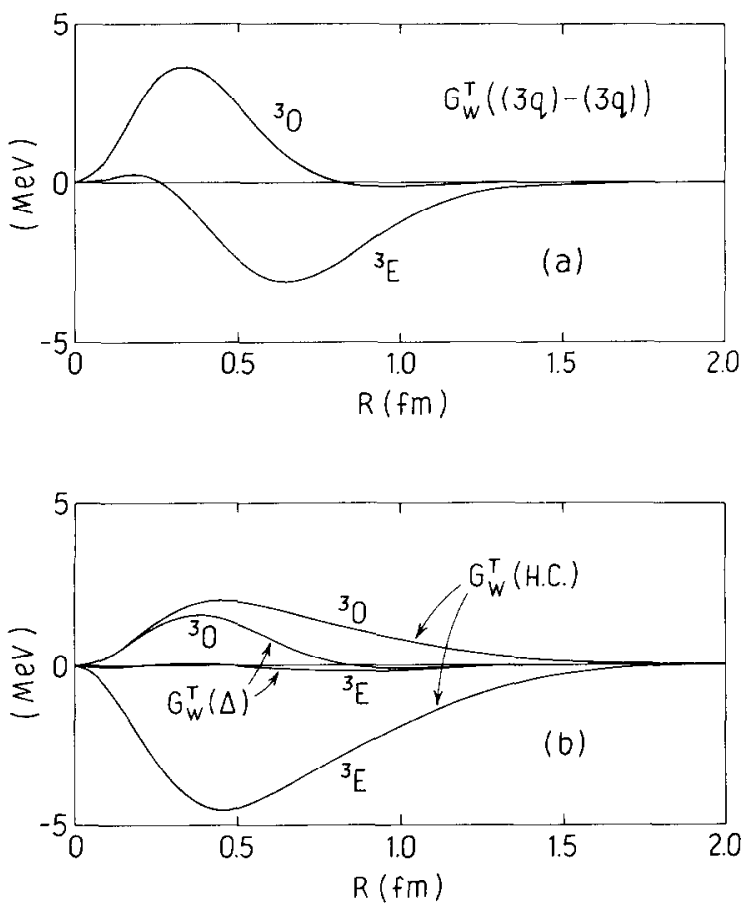

Fig. 1. (a) The contributions of the pure (3q)-(3q) part of the kernel to the Wigner transform of the tensor term at $P=0, c_{0}^{4} G_{0 \mathrm{~W}}^{T}(R, 0)$. (b) Contributions of the $(3 q)(\mathrm{q} \overline{\mathrm{q}})$ coupling kernels to the Wigner transform of the tensor term at $P=0$, as defined by eq. (14a); (HC) includes the sum over the 15 terms $\alpha$ of hidden color type, $(\Delta)$ includes the sum over terms $\alpha$ with $\Delta \rho \frac{1}{2}, \Delta \pi \frac{3}{2}, \Delta \rho \frac{3}{2}$. Note that the simple ( $\mathrm{q} \overline{\mathrm{q}})$ exchange terms make no contribution to these channels.

fact that the coefficients $c_{\Delta \pi 3 / 2}, c_{\Delta \rho 3 / 2}$, and $c_{\Delta \rho 1 / 2}$ are among the largest $c_{\alpha}$ of our model [see table 1 of ref. ${ }^{1}$ )]. Figs. 2a and $b$ show the contributions of the $\mathrm{N} \eta$ and $\mathrm{N} \omega$ coupling kernels. They are not only negligible but also cancel each other. Both are properties shared with the $\eta$ and $\omega$ tensor force terms of conventional meson exchange potentials.

As for the conventional OBEP's, therefore, the tensor force of our quark model arises almost completely through $\mathrm{N} \pi$ and $\mathrm{N} \rho$ terms. These are shown in figs. 3 and 4. Since the pion-nucleon coupling constant predicted by our model is too small by a factor of $\sim 3$, the $\mathbf{N} \pi$ tensor force must be increased to attain a realistic value. Since specific pieces of the interaction can be isolated and improved in our model it is straightforward to effect this change. The improvement in the $\mathrm{N} \pi$ force has been made in two ways. In the first, shown through the solid and dashed curves of fig. 3 , the strength of the $\mathrm{N} \pi$ tensor component of the Wigner transform has been increased by a factor of 3 . In the second (the crossed curve), the $N \pi$ tensor force has been replaced with a pion tensor term (OPEP) calculated with a $g_{\mathrm{NN} \pi}^{2}(0)=14.17$ and the form factor $g^{2}(k)$ predicted by our quark model, see eq. (69) of ref. ${ }^{1}$ ). This 

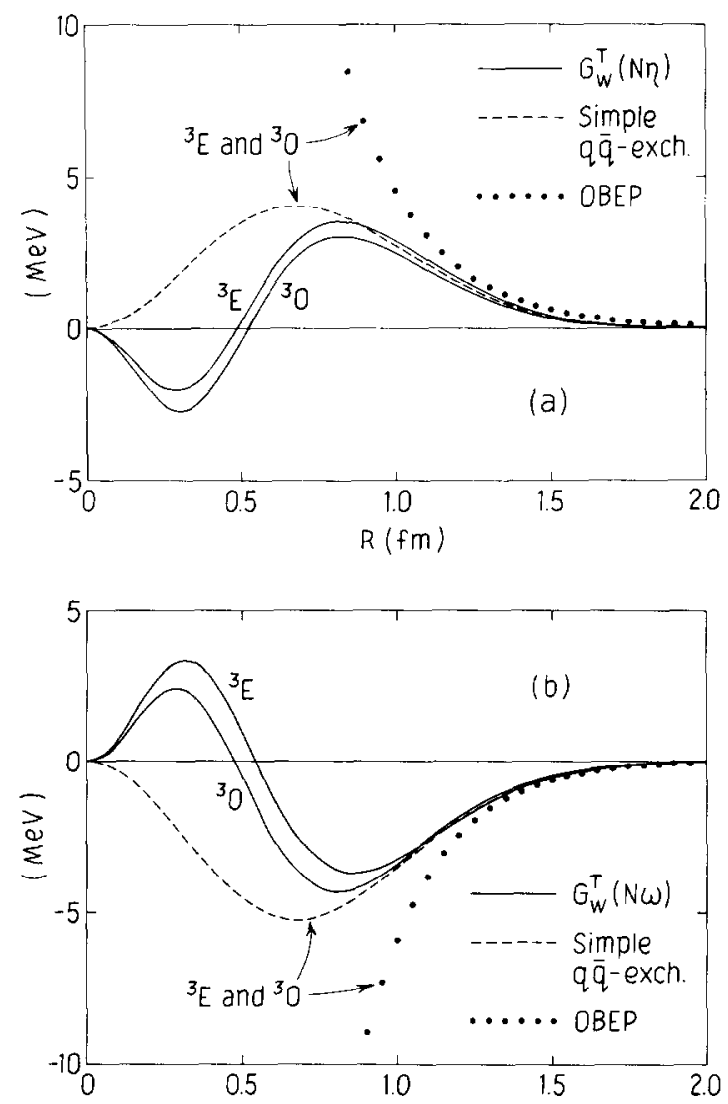

Fig. 2. Comparison of the $\boldsymbol{P}=0$ Wigner transforms, as defined by eq. (14a), with the predicted OBEP's (dotted curves). Solid lines give the contributions of all 25 types of exchange terms. Dashed lines give contributions of the simple ( $\mathrm{q} \bar{q})$ exchange terms only; i.e. $P=(37)$ and $i=1,2$, and 3 terms only. For the details of the OBEP's, see ref. ${ }^{3}$ ). (a) Tensor part of type $\alpha=\mathrm{N} \eta$. (b) Tensor part of type $\alpha=\mathrm{N} \omega$.

predicted $\boldsymbol{k}$-dependence is approximated extremely well by a simple gaussian function $g^{2}(k)=14.17 \exp \left[-0.094 k^{2}\right]$. Fig. 3 shows that the latter gives the best approximation to a conventional OPEP tensor force in the extreme long range limit; but the former, which is the closest to the full spirit of our quark model, gives the better approximation to the conventional OPEP in the 0.8 to $1.2 \mathrm{fm}$ region where the OPEP tensor force has its largest value. For this reason it is this form of the improved $\mathrm{N} \pi$ term which is used for the demonstration of the full tensor force in fig. 5. The solid curves of fig. 5 give the full Wigner transforms of the tensor terms including the contributions of the pure (3q)-(3q) kernels, $G_{0}$, and all 24 components of the (3q)(q̄) coupling kernels, $G_{\alpha}$, where the full set of 25 possible exchange terms has been retained. The dashed curves on the other hand give only the contributions of the simple ( $\mathrm{q} \overline{\mathrm{q}}$ ) exchange terms of the coupling kernels. These give a remarkably good approximation to the full potentials. The quark model predictions are also in 


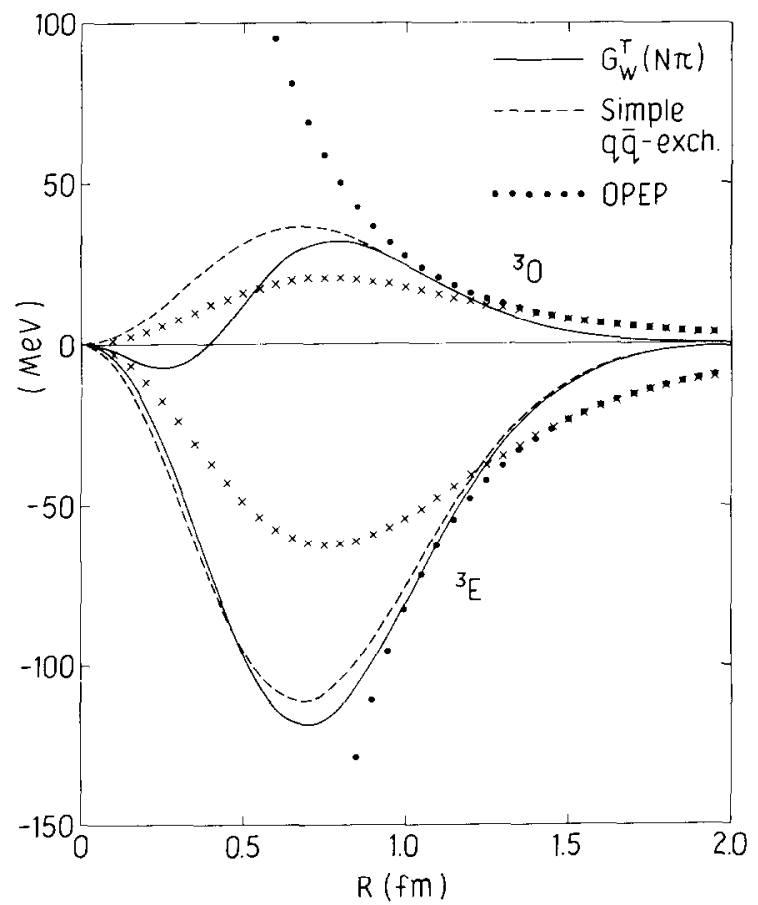

Fig. 3. Same as for fig. 2, with $\alpha=N \pi$; but note the change of energy scale from fig. 2. Improved $N \pi$ Wigner transforms are used. In the solid and dashed curves $G_{\mathrm{W}}^{\mathrm{N} \pi T}$ has been increased by a factor of 3 as required by the observed pion-nucleon coupling constant. The crossed curves show the OPEP tensor force with gaussian form factor as predicted by our quark model, with $g_{\mathrm{NN} \pi}^{2}(0)=14.17$, see text.

reasonable agreement with conventional OBEP tensor terms over the important $R>0.8 \mathrm{fm}$ region.

At extremely short range the quark model tensor terms go to zero. This is consistent with a recent attempt to combine a meson exchange potential with a short range repulsive core derived from a pure ( $3 q)-(3 q)$ model to give a good account of the low energy NN data ${ }^{13}$ ). However, in ref. ${ }^{13}$ ) the short range cutoff factors are put in by hand, while in the present study the regular behavior of the potentials in the short range region is a natural consequence of the quark model itself.

As already shown in refs. ${ }^{5,14}$ ) the pure $(3 q)-(3 q)$ kernels lead to significant spin-orbit forces. These have their origin in the spin-orbit terms of the one-gluon exchange Breit interaction, but additional spin-orbit terms may be generated by the confinement mechanism. The uncertainties associated with spin-orbit terms generated by different quark confinement mechanisms were discussed in ref. ${ }^{5}$ ). Due to a sign error in the antisymmetric spin-orbit terms, kowever, this uncertainty was magnified [see the erratum of ref. ${ }^{5}$ )]. If the confinement potential arises through scalar coupling, $(\bar{\Psi} \Psi)$, the additional spin-orbit terms generated by such a confining mechanism vanish completely if the confining potential is quadratic, and they are 


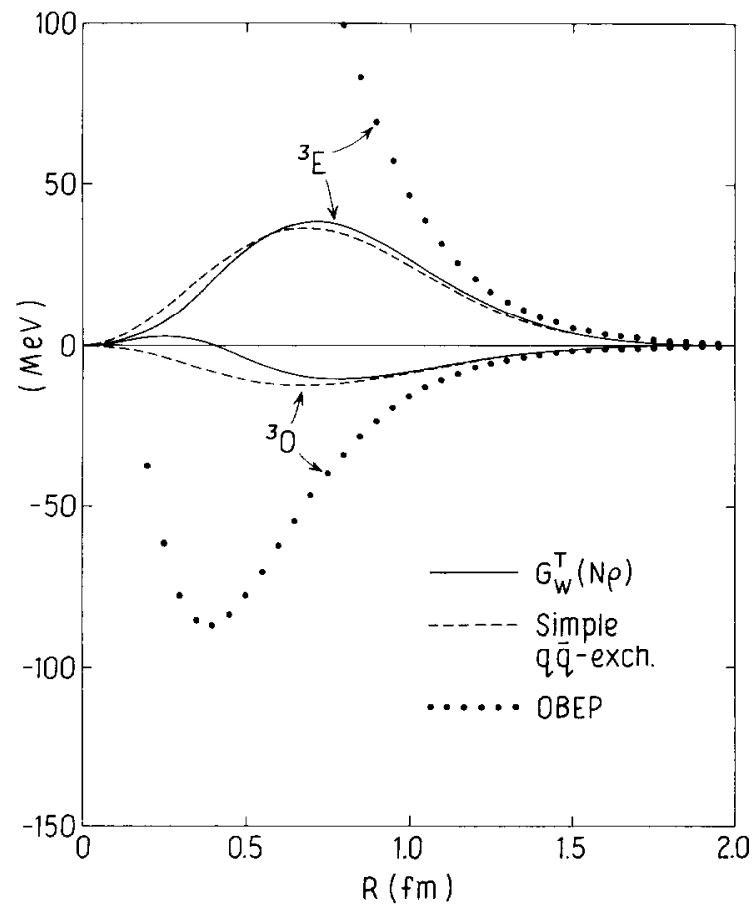

Fig. 4. Same as for fig. 2, with summed contributions from $\alpha=N \rho \frac{1}{2}$ and $N \rho \frac{3}{2}$.

essentially negligible for linear confining potentials ${ }^{5}$ ). This type of spin-orbit term thus shares the gencral property of our quark model of being almost completely independent of the strength of the confinement potential. However, both the symmetric and antisymmetric spin-orbit terms of the Breit quark-gluon interaction give significant contributions to the $L S$ exchange kernels of pure (3q)-(3q) type. Their Wigner transforms at $\boldsymbol{P}=0$ are shown in fig. 6. Comparison with ref. ${ }^{5}$ ) (bearing in mind the value of $c_{0}^{4} \sim 0.5$ ) shows that such potentials are quite insensitive to rather large changes in the model parameters.

As for the tensor terms, the most important contributions to the $L S$ potentials come from the $(3 q)(q \bar{q})$ coupling kernels. This is shown in fig. 7 which gives both the full $L S$ potential and the separate contributions of the $(3 q)(\mathrm{q} \overline{\mathrm{q}})$ coupling kernels including all 24 components, $\alpha$, and all 25 exchange terms. In the important 0.7 to $1.0 \mathrm{fm}$ region, e.g., about $60-65 \%$ of the triplet-odd $L S$ potential results from the $(3 q)(q \bar{q})$ coupling kernels. The most important contributors to these coupling kernels come from $\mathrm{N} \rho$ and $\mathrm{N} \omega$ components. This is again a property shared with the $L S$ terms of conventional OBEP's. Figs. 8 and 9 show the Wigner transforms, at $\boldsymbol{P}=\mathbf{0}$, for the $N \rho$ and $N \omega$ components. The solid curves give the contributions of all 25 exchange types. Except for the extreme short range part of the triplet-odd $\mathrm{N} \omega$ term these are also approximated very well by the simple $(\mathrm{q} \overline{\mathrm{q}})$ exchange terms; that is 


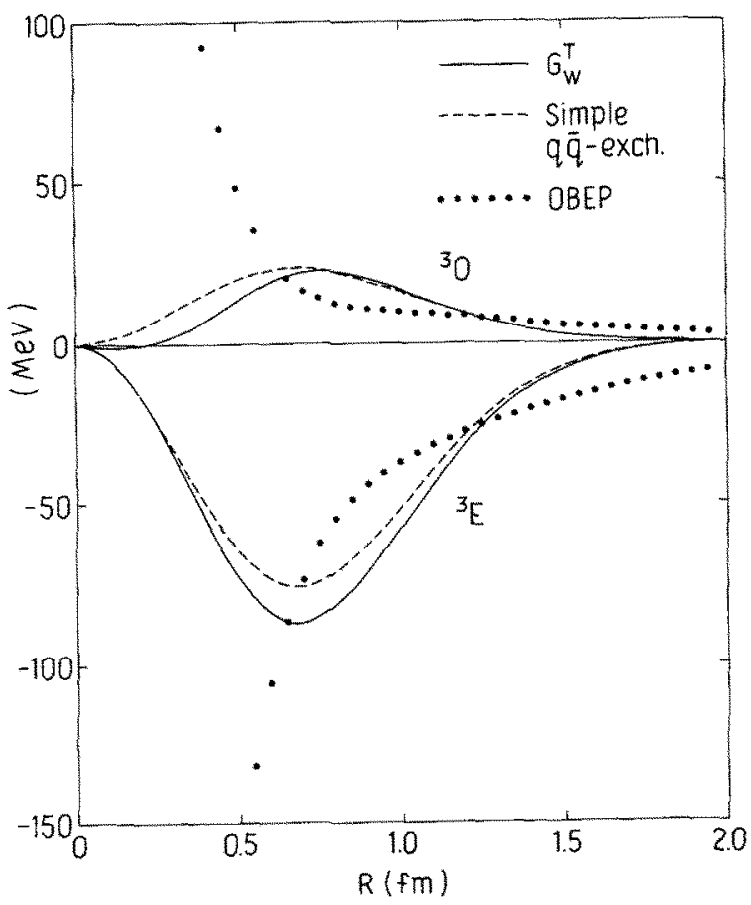

Fig. 5. The full tensor force. The solid curves give the contributions to the Wigner transform at $P=0$ of the pure ( $3 q)-(3 q)$ term and all $24(3 q)(q \bar{q})$ coupling kernels including all 25 exchange terms for each $\alpha$. The dashed curves give the simple (qव) exchange contributions of the coupling kernels. The dotted OBEP curves include $\eta, \pi, \rho, \omega$, and the very small $\phi$ contribution.

by only two of the 25 possible exchange terms. The $N \eta$ and $N \pi$ components (not shown in the figure) make negligible contributions to the $L S$ potentials, with the possible exception of the short range part of the triplet-odd $\mathrm{N} \pi$ term with a Wigner transform which ranges from $-15 \mathrm{MeV}$ at $0.7 \mathrm{fm}$ to $-64 \mathrm{MeV}$ at $0.5 \mathrm{fm}$ and to $-297 \mathrm{MeV}$ at $R=0$. Fig. 10 shows that the contributions of the many $(3 q)(q \bar{q})$ hidden color channels and the coupling terms with ( $3 q)$ pieces of $\Delta$-type may make significant contributions to the $L S$ potentials. However, these are of extremely short range, and the hidden color pieces partially cancel the $\Delta$ contributions.

In the important $R>0.7 \mathrm{fm}$ range the $L S$ potentials of our quark model therefore have most of the characteristics of conventional meson exchange spin-orbit potentials. Fig. 7 also shows that the $L S$ potentials derived from our quark model are in remarkably good quantitative agreement with conventional OBEP $L S$ potentials in this long-range limit.

\section{Summary}

The present study focuses on the noncentral parts of the NN interaction since these can be expected to be the most reliable at the present stage of development 


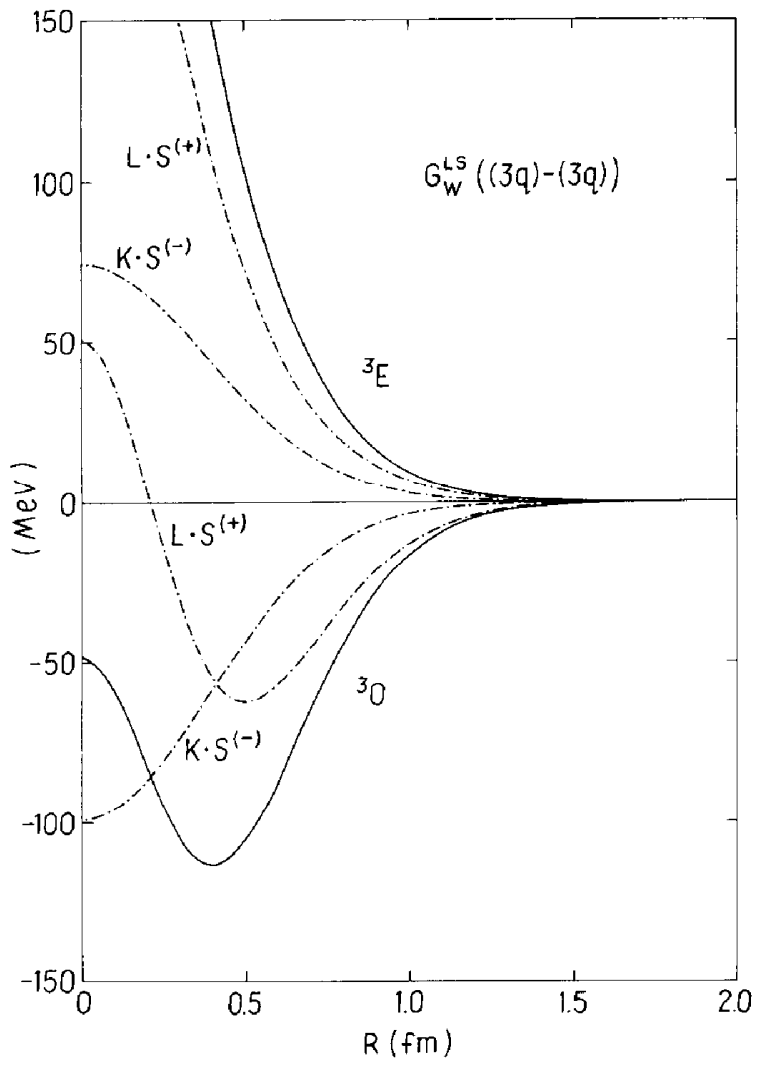

Fig. 6. The contribution of the pure (3q)-(3q) part of the kernel to the Wigner transform of the $L S$ term, $c_{0}^{4} G_{0 \mathrm{w}}^{L S}(R, 0)$. The dash-dotted curves show the separate contributions of the symmetric, $L \cdot S^{(+)}$, and antisymmetric, $\boldsymbol{K} \cdot \boldsymbol{S}^{(-)}$, spin-orbit terms.

of our quark model, in which the $(q \bar{q})$ and $(q \bar{q})^{2}$ excitations inherent in the quark-gluon interaction have been explicitly incorporated into the model space. The tensor force arises almost exclusively from the $(3 q)(q \bar{q})$ coupling kernels, and despite their opposite signs, gains almost its full strength from the $\mathrm{N} \pi$ and $\mathrm{N} \rho$ coupling kernels, a property shared with conventional meson exchange potentials.

If the $\mathrm{N} \pi$ contribution is adjusted to fit the experimental nucleon-pion coupling constant, the predicted strength of the tensor force is also in reasonable agreement with that of conventional OBEP's over the range for which the $L \geqslant 1$ partial wave relative motion functions permit the tensor force to act. Finally, the tensor force derived from the full exchange kernel is approximated extremely well by the simple $(q \bar{q})$ exchange terms, another property pointing to the essentially mesonic character of this exchange force. Although the $L S$ force gains significant contributions from the pure (3q)-(3q) sector, it is again dominated by the $(3 q)(q \bar{q})$ coupling kernels. In the 0.7 to $1.0 \mathrm{fm}$ range about $60-65 \%$ of the triplet odd $L S$ potential results from the $(3 q)(q \bar{q})$ coupling kernels. The dominant contributions come from the $N \rho$ and 


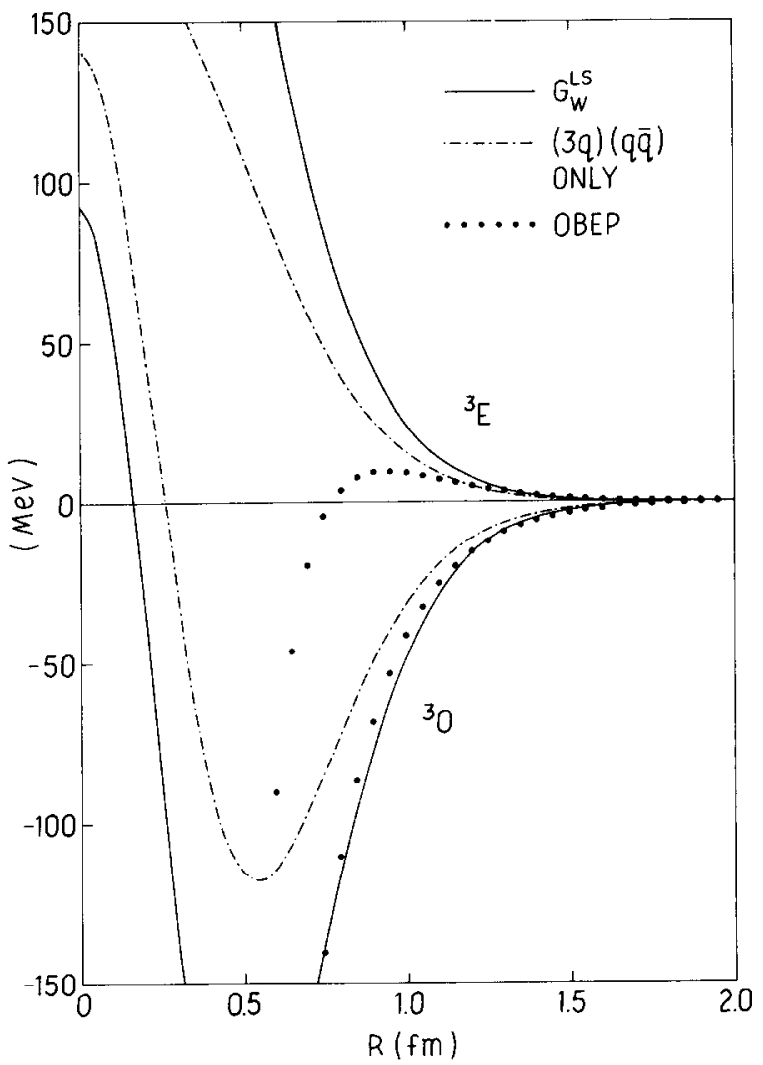

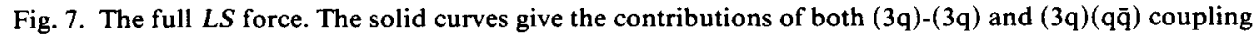
kernels. (The minimum for the ${ }^{3} \mathrm{O}$ potential falls at $-221 \mathrm{MeV}$ ). The dash-dotted curves are for the $(3 q)(q \bar{q})$ coupling terms only. The dotted OBEP curves include $\rho, \omega, \sigma$, and very small $\phi, \delta$ contributions.

$\mathrm{N} \omega$ components, again a property shared with conventional meson exchange potentials. Fig. 8 shows that the $\mathrm{N} \rho$ component in the important triplet-odd state is in remarkably good agreement with the corresponding OBEP in the region where $L \geqslant 1$ partial wave functions permit the $L S$ force to act. The agreement for the $\mathrm{N} \omega$ component (fig. 9), is not quite as good. However, the pure (3q)-(3q) contribution seems to compensate for this small deficiency, so that the $L S$ potentials derived from the full exchange kernel of our quark model are again in remarkably good agreement with the full OBEP $L S$ potentials (fig. 7).

Finally, to test the absolute values of our predicted noncentral forces, fig. 11 shows the RGM phase shifts predicted by the noncentral kernels of our model for the ${ }^{3} \mathrm{P}$ partial waves. Since conventional discussions of the $\mathrm{NN}$ interaction lead to very weak triplet-odd central terms in the long range region, the ${ }^{3} \mathrm{P}$ phase shifts arise almost exclusively through the tensor and $L S$ terms. For this reason the triplet-odd central terms of our quark model have been turned off for the calculation 

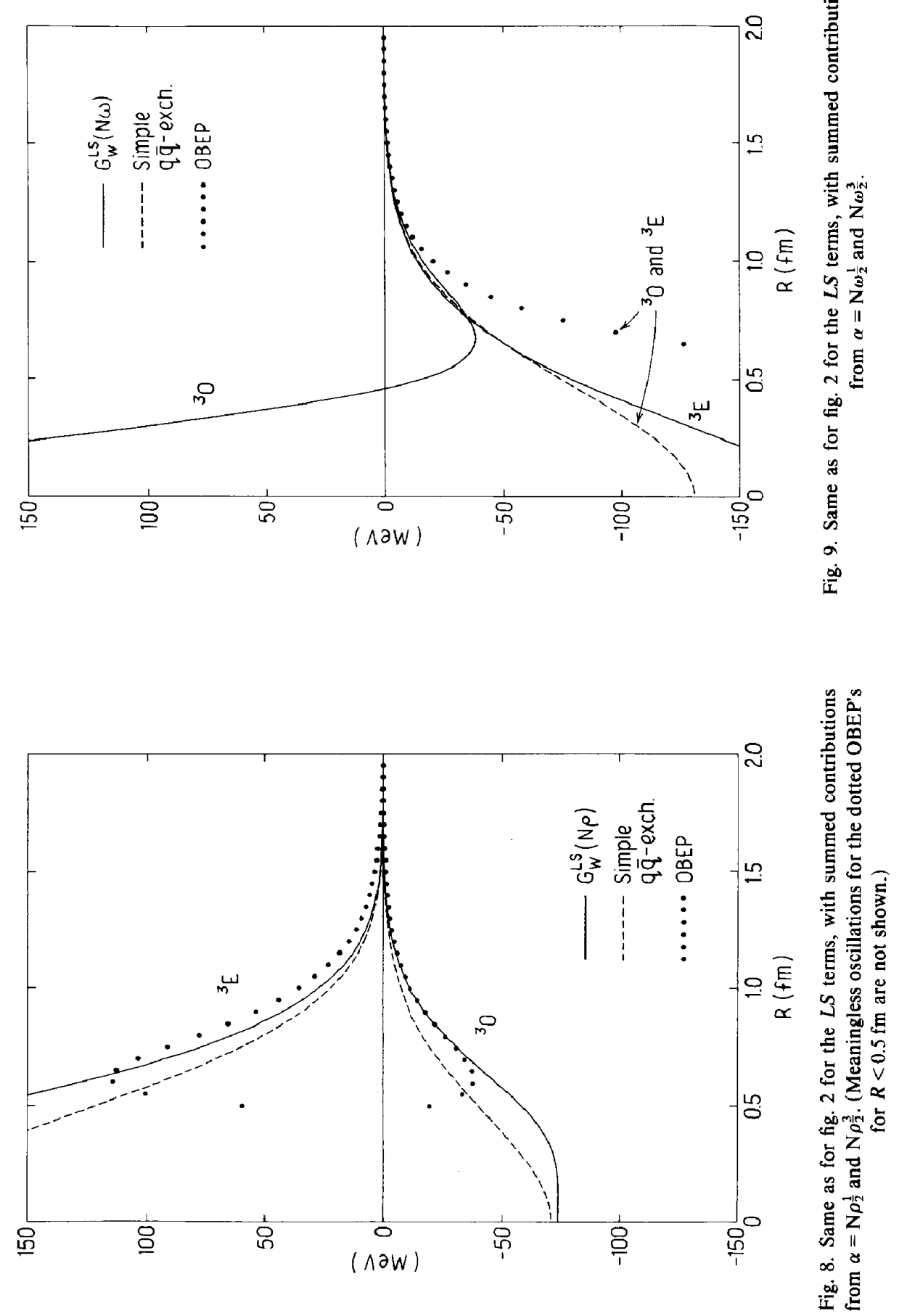


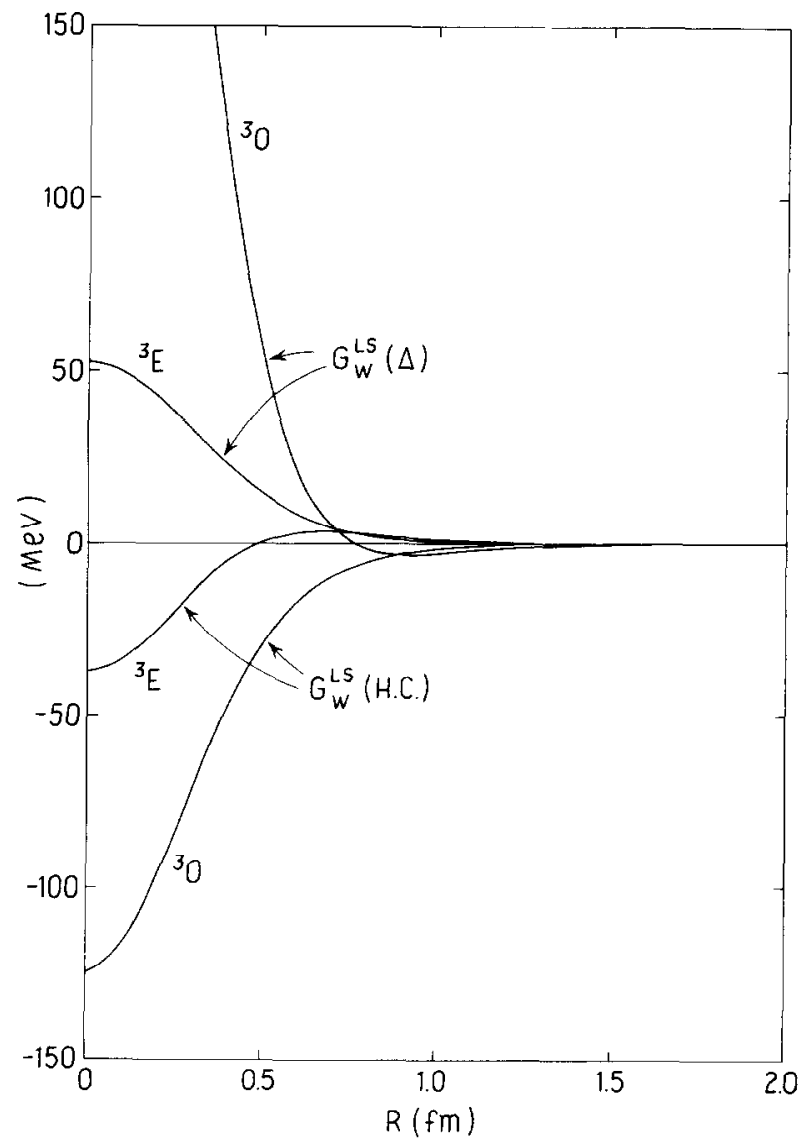

Fig. 10. Contributions of the (3q)(qव) coupling kernels to the Wigner transform of the $L S$ term at $P=0$, as defined by eq. (14a). (HC) includes the sum over the 15 terms $\alpha$ of hidden color type; $(\Delta)$ includes the sum over terms $\alpha$ with $\Delta \rho \frac{1}{2}, \Delta \pi \frac{3}{2}, \Delta \rho \frac{3}{2}$.

of ${ }^{3} \mathrm{P}$ phase shifts, and fig. 11 gives the results derived from pure noncentral kernels. Since the long range part of the pion tensor force is crucial for the extreme low energy ${ }^{3} \mathrm{P}_{0}$ phase shift, the improved $\mathrm{N} \pi$ tensor term chosen for our quark model is that illustrated by the crossed curve of fig. 3 , in which the adjusted gaussian form factor with $\boldsymbol{k}$-dependence predicted by our quark model has been used; (i.e. $\left.g_{\mathrm{NN} \pi}^{2}(k)=14.17 \exp \left(-0.094 k^{2}\right)\right)$. Except for this adjustment of the $\mathrm{N} \pi$ tensor term, however, these ${ }^{3} \mathrm{P}$ partial wave predictions are completely free of parameter fits since the parameters of our model have been determined by single nucleon properties. The attractive low energy ${ }^{3} \mathrm{P}_{0}$ phase shift is dominated by the tensor force while its higher energy repulsive character arises through the $L S$ force. The ${ }^{3} P_{1}$ phase shift, on the other hand, gets significant repulsive contributions from both tensor and $L S$ terms, while the attractive character of the ${ }^{3} \mathrm{P}_{2}$ phase shift arises through the $L S$ force with weaker tensor contributions. The good fit of the predicted values thus 


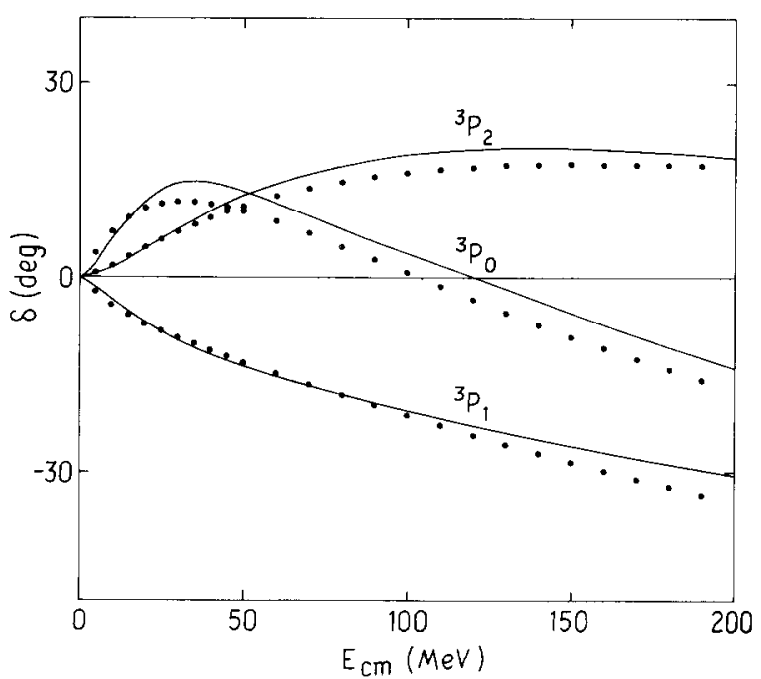

Fig. 11. The ${ }^{3} \mathrm{P}$ phase shifts predicted by the pure noncentral kernels. The solid curves give the results of RGM calculations including the very small effect of ${ }^{3} \mathrm{P}_{2}-{ }^{3} \mathrm{~F}_{2}$ channel coupling. The triplet-odd central terms have been turned off (see text). The (dotted) experimental points are taken from MacGregor $e t$ al., Phys. Rev. 182 (1969) 1714.

indicates that both the tensor and spin-orbit forces of our quark model are in good agreement with the experimental facts.

\section{Appendix A}

In this appendix, we will consider the purely mathematical problem of how to convert the complex GCM kernels to the standard RGM form. We will also construct the Wigner transforms of the RGM kernels. Although the formulas given here are tailored to the GCM coupling kernels of sect. 2, they may also be very useful in other nuclear cluster problems. In particular, the $L S$ and tensor kernels of the (3q)-(3q) system ${ }^{5}$ ) are easily obtained through a simple special case of these formulas. The evaluation of RGM kernels is a first step in the calculation of RGM matrix elements in analytic form.

Suppose $I^{\mathrm{GCM}}\left(z, z^{\prime}\right)$ is one specific term of a gaussian complex GCM kernel for a two-cluster system. The RGM form of this term is obtained through the Bargmann transformation

$$
I\left(\boldsymbol{R}, \boldsymbol{R}^{\prime}\right)=\int \mathrm{d} \mu(z) \mathrm{d} \mu\left(z^{\prime}\right) A_{\gamma_{1}}(\boldsymbol{R}, z) A_{\gamma_{0}}\left(\boldsymbol{R}^{\prime}, z^{\prime}\right)^{*} I^{\mathrm{GCM}}\left(z, z^{\prime}\right),
$$

where

$$
A_{\gamma}(\boldsymbol{R}, z)=\left(\frac{2 \gamma}{\pi}\right)^{3 / 4} \exp \left[-\gamma\left(\boldsymbol{R}-\frac{z}{\sqrt{\gamma}}\right)^{2}+\frac{1}{2} z^{2}\right]
$$


Here, $z, z^{\prime}$ are 3 dimensional Bargmann space variables, and the 6 dimensional $\mathrm{d} \mu(z)$ contains the Bargmann weighting factor $\left.\pi^{-3} \exp \left(-z^{*} \cdot z\right)^{6,7}\right)$. The Wigner transform of $I\left(R, R^{\prime}\right)$ is defined by

$$
G_{\mathrm{W}}(\boldsymbol{R}, \boldsymbol{P})=\int \mathrm{d} s \exp \left[\frac{i}{\hbar}(s \cdot \boldsymbol{P})\right]\left\{\begin{array}{c}
I\left(\boldsymbol{R}-\frac{1}{2} s, \boldsymbol{R}+\frac{1}{2} s\right) \\
I\left(\boldsymbol{R}-\frac{1}{2} s,-\boldsymbol{R}-\frac{1}{2} s\right)
\end{array}\right\},
$$

where the upper/lower case applies according to whether the coefficient of the $\left(\boldsymbol{R} \cdot \boldsymbol{R}^{\prime}\right)$ term in the exponential factor of $I\left(\boldsymbol{R}, \boldsymbol{R}^{\prime}\right)$ is positive/negative $\left.{ }^{10}\right)$.

A simple formula for $I\left(\boldsymbol{R}, \boldsymbol{R}^{\prime}\right)$ or $G_{\mathrm{W}}(\boldsymbol{R}, \boldsymbol{P})$ is well known if $I^{\mathrm{GCM}}\left(z, z^{\prime}\right)$ is a pure gaussian kernel [e.g., see table 1 of ref. $^{11}$ ) and appendix $B$ of ref. $\left.{ }^{9}\right)$ ]; viz., if $I^{\mathrm{GCM}}\left(z, z^{\prime}\right)$ is

$$
I_{\exp }^{\mathrm{GCM}}\left(z, z^{\prime}\right)=\exp \left[-\frac{1}{2} f z^{* 2}-\frac{1}{2} g z^{\prime 2}+h\left(z^{*} \cdot z^{\prime}\right)\right],
$$

then, the RGM form is

$$
I_{\text {exp }}\left(\boldsymbol{R}, \boldsymbol{R}^{\prime}\right)=\left(\frac{2 \sqrt{\gamma_{1} \gamma_{0}}}{\pi D}\right)^{3 / 2} \exp \left[-A \gamma_{1} \boldsymbol{R}^{2}-B \gamma_{0} \boldsymbol{R}^{\prime 2}+C \sqrt{\gamma_{1} \gamma_{0}}\left(\boldsymbol{R} \cdot \boldsymbol{R}^{\prime}\right)\right]
$$

with

$$
\begin{gathered}
D=(1-f)(1-g)-h^{2}, \\
A=\frac{2(1-g)}{D}-1, \quad B=\frac{2(1-f)}{D}-1, \quad C=\frac{4 h}{D} .
\end{gathered}
$$

On the other hand, the Wigner transform is given by

$$
G_{\mathrm{W}}^{\exp }(\boldsymbol{R}, \boldsymbol{P})=\left(\frac{4}{D_{\mathrm{w}}}\right)^{3 / 2} \exp \left[-\eta\left(\frac{\boldsymbol{R}}{b}\right)^{2}-\zeta\left(\frac{b \boldsymbol{P}}{\hbar}\right)^{2}+i \xi\left(\frac{\boldsymbol{R} \cdot \boldsymbol{P}}{\hbar}\right)\right]
$$

with

$$
\begin{aligned}
D_{\mathrm{w}} & =\bar{\mu}\left(h^{2}-f g+1\right)+\tilde{\mu}(f-g)+s(h) 2 h, \\
\eta & =\left(\mu_{1} \mu_{0}\right)^{1 / 2}\left[(1+f)(1+g)-h^{2}\right] / D_{\mathrm{w}}, \\
\zeta & =\left(\mu_{1} \mu_{0}\right)^{-1 / 2}\left(D / D_{\mathrm{w}}\right), \\
\xi & =2\left[\tilde{\mu}\left(h^{2}-f g+1\right)+\bar{\mu}(f-g)\right] / D_{\mathrm{w}},
\end{aligned}
$$

where $s(h)$ is the sign of $h, s(h)=h /|h|$, and the reduced mass numbers $\mu_{1}, \mu_{0}, \bar{\mu}$, and $\tilde{\mu}$ are introduced through

$$
\begin{gathered}
\gamma_{i}=\mu_{i} / 2 b^{2} \quad(i=0,1), \\
\bar{\mu}=\left(\mu_{1}+\mu_{0}\right) / 2 \sqrt{\mu_{1} \mu_{0}}, \quad \tilde{\mu}=\left(\mu_{1}-\mu_{0}\right) / 2 \sqrt{\mu_{1} \mu_{0}} .
\end{gathered}
$$

If the cartesian components of the polynomial terms are introduced in the GCM kernel by

$$
I_{j k}^{\mathrm{GCM}}\left(z, z^{\prime}\right)=I_{\exp }^{\mathrm{GCM}}\left(z, z^{\prime}\right)\left[\left(a z^{*}+b z^{\prime}\right)_{j}\left(c z^{*}+d z^{\prime}\right)_{k}+e \delta_{j k}\right]
$$


then the central, $L S$, and tensor RGM kernels are defined through

$$
\begin{aligned}
\mathcal{M}^{\mathrm{C}}\left(\boldsymbol{R}, \boldsymbol{R}^{\prime}\right)= & \frac{1}{3} \sum_{j} I_{j j}\left(\boldsymbol{R}, \boldsymbol{R}^{\prime}\right), \\
\mathcal{M}^{L S}\left(\boldsymbol{R}, \boldsymbol{R}^{\prime}\right)= & i \sum_{i j k} \varepsilon_{i j k} I_{j k}\left(\boldsymbol{R}, \boldsymbol{R}^{\prime}\right) S_{i}, \\
\mathcal{M}^{\mathrm{T}}\left(\boldsymbol{R}, \boldsymbol{R}^{\prime}\right)= & \frac{1}{2} \sum_{j k}\left[I_{j k}\left(\boldsymbol{R}, \boldsymbol{R}^{\prime}\right)+I_{k j}\left(\boldsymbol{R}, \boldsymbol{R}^{\prime}\right)\right] \sigma_{1 j} \sigma_{2 k} \\
& -\mathcal{M}^{\mathrm{C}}\left(\boldsymbol{R}, \boldsymbol{R}^{\prime}\right)\left(\boldsymbol{\sigma}_{1} \cdot \boldsymbol{\sigma}_{2}\right),
\end{aligned}
$$

where $I_{j k}\left(\boldsymbol{R}, \boldsymbol{R}^{\prime}\right)$ is the RGM form of $I_{j k}^{\mathrm{GCM}}\left(z, z^{\prime}\right)$. It is convenient to separate the RGM kernels $\mathcal{M}^{\Omega}\left(\boldsymbol{R}, \boldsymbol{R}^{\prime}\right)$ and their Wigner transforms $G_{\mathrm{w}}^{\Omega}(\boldsymbol{R}, \boldsymbol{P})$ (for $\Omega=\mathrm{C}, L S$, $T)$, into exponential and polynomial factors,

$$
\begin{aligned}
& \mathcal{M}^{\Omega}\left(\boldsymbol{R}, \boldsymbol{R}^{\prime}\right)=I_{\exp }\left(\boldsymbol{R}, \boldsymbol{R}^{\prime}\right) P^{\Omega}\left(\boldsymbol{R}, \boldsymbol{R}^{\prime}\right), \\
& G_{\mathrm{W}}^{\Omega}\left(\boldsymbol{R}, \boldsymbol{R}^{\prime}\right)=G_{\mathrm{W}}^{\exp }(\boldsymbol{R}, \boldsymbol{P}) P_{\mathrm{w}}^{\Omega}(\boldsymbol{R}, \boldsymbol{P}),
\end{aligned}
$$

where the polynomial factors are given by

$$
\begin{aligned}
P^{\mathrm{C}}\left(\boldsymbol{R}, \boldsymbol{R}^{\prime}\right) & =E+\frac{2}{3}\left(\boldsymbol{V}_{a b} \cdot \boldsymbol{V}_{c d}\right), \\
P^{L S}\left(\boldsymbol{R}, \boldsymbol{R}^{\prime}\right) & =V^{L S} \sqrt{\gamma_{1} \gamma_{0}} i\left(\left[\boldsymbol{R} \times \boldsymbol{R}^{\prime}\right] \cdot \boldsymbol{S}\right), \\
P^{\mathrm{T}}\left(\boldsymbol{R}, \boldsymbol{R}^{\prime}\right) & =\frac{2}{3} S_{12}\left(\boldsymbol{V}_{a b}, \boldsymbol{V}_{c d}\right) \\
P_{\mathrm{W}}^{\mathrm{C}}(\boldsymbol{R}, \boldsymbol{P}) & =E_{\mathrm{W}}+\frac{2}{3}\left(\boldsymbol{V}_{a b}^{\mathrm{W}} \cdot V_{c d}^{\mathrm{w}}\right), \\
P_{\mathrm{W}}^{L S}(\boldsymbol{R}, \boldsymbol{P}) & =V_{\mathrm{W}}^{L S} \frac{1}{\hbar}([\boldsymbol{R} \times \boldsymbol{P}] \cdot \boldsymbol{S}), \\
P_{\mathrm{W}}^{\mathrm{T}}(\boldsymbol{R}, \boldsymbol{P}) & =\frac{2}{3} S_{12}\left(\boldsymbol{V}_{a b}^{\mathrm{w}}, \boldsymbol{V}_{c d}^{\mathrm{W}}\right) .
\end{aligned}
$$

The tensor operators $S_{12}(U, V)$ are defined in eq. (13). The coefficients $E, V^{L S}$, and the vectors $V_{a b}\left(V_{c d}\right)$ in (A.13) are conveniently expressed as

$$
\begin{gathered}
E=e-(H / D), \quad V^{L S}=(4 / D)(a d-b c), \\
V_{a b}=\left[F_{a b} \sqrt{2 \gamma_{1}} R+G_{a b} \sqrt{2 \gamma_{0}} R^{\prime}\right] / D,
\end{gathered}
$$

with

$$
\begin{gathered}
F_{a b}=(1-g) a-h b, \quad G_{a b}=(1-f) b-h a, \\
H=F_{a b} c+G_{a b} d=F_{c d} a+G_{c d} b .
\end{gathered}
$$

Similarly, for the Wigner transform, one finds

$$
\begin{aligned}
E_{\mathrm{W}} & =e-\left(H_{\mathrm{W}} / D_{\mathrm{W}}\right), \quad V_{\mathrm{W}}^{L S}=-s(h)\left(4 / D_{\mathrm{W}}\right)(a d-b c), \\
\boldsymbol{V}_{a b}^{\mathrm{W}} & =\left[F_{a b}^{\mathrm{W}}\left(\mu_{1} \mu_{0}\right)^{1 / 4}\left(\frac{\boldsymbol{R}}{b}\right)+G_{a b}^{\mathrm{W}}\left(\mu_{1} \mu_{0}\right)^{-1 / 4} i\left(\frac{b P}{\hbar}\right)\right] / D_{\mathrm{W}},
\end{aligned}
$$


with

$$
\begin{aligned}
& F_{a b}^{\mathrm{W}}=\left(\frac{\mu_{0}}{\mu_{1}}\right)^{1 / 4}[(1+g) a+h b]+s(h)\left(\frac{\mu_{1}}{\mu_{0}}\right)^{1 / 4}[(1+f) b+h a], \\
& G_{a b}^{\mathrm{W}}=s(h)\left(\frac{\mu_{0}}{\mu_{1}}\right)^{1 / 4}[(1-f) b-h a]-\left(\frac{\mu_{1}}{\mu_{0}}\right)^{1 / 4}[(1-g) a-h b], \\
& H_{\mathrm{W}}=(\bar{\mu} g-\tilde{\mu}) a c+(\bar{\mu}+\tilde{\mu}) b d+(\bar{\mu} h+s(h))(a d+b c) .
\end{aligned}
$$

If a gaussian function $u(r)=\exp \left(-\chi^{2} r^{2}\right)$ is used for the local two-body interaction, the GCM kernel in eq. (A.4) has the general structure

$$
I_{\mathrm{exp}}^{\mathrm{GCM}}\left(z, z^{\prime}\right)=I_{\exp }^{(0) \mathrm{GCM}}\left(z, z^{\prime}\right) \exp \left[-\frac{1}{2} \lambda\left(p z^{*}+q z^{\prime}\right)^{2}\right] \text {, }
$$

where $I_{\exp }^{(0) G C M}\left(z, z^{\prime}\right)$ is defined through eq. (A.4) with the coefficients $f, g, h$ replaced by $f^{(0)}, g^{(0)}, h^{(0)}$. In eq. (A.19), $\lambda$ is a simple function of $\chi^{2}(\lambda=\kappa /(6+\kappa)=$ $b^{2} \chi^{2} /\left(6+13 b^{2} \chi^{2}\right)$ in the main text), and the parameters $p, q$ depend on the nature of the exchange interaction kernel [see eq. (11) of the main text]. Since the parameters $f, g, h$ are now parametrized as

$$
f=f^{(0)}+\lambda p^{2}, \quad g=g^{(0)}+\lambda q^{2}, \quad h=h^{(0)}-\lambda p q,
$$

the formulas (A.4)-(A.9) admit the following representations for $I_{\text {exp }}\left(\boldsymbol{R}, \boldsymbol{R}^{\prime}\right)$ and $G_{\mathrm{W}}^{\exp }(\boldsymbol{R}, \boldsymbol{P})$;

$$
\begin{aligned}
& I_{\text {exp }}\left(\boldsymbol{R}, \boldsymbol{R}^{\prime}\right)=I_{\exp }^{(0)}\left(\boldsymbol{R}, \boldsymbol{R}^{\prime}\right)\left[\frac{1}{1-\lambda \alpha}\right]^{3 / 2} \exp \left[-\frac{\lambda}{1-\lambda \alpha}\left(\boldsymbol{V}_{p \mathbf{q}}^{(0)}\right)^{2}\right] \\
& G_{\mathrm{W}}^{\exp }(\boldsymbol{R}, \boldsymbol{P})=G_{\mathrm{W}}^{\exp (0)}(\boldsymbol{R}, \boldsymbol{P})\left[\frac{1}{1-\lambda \alpha_{\mathrm{W}}}\right]^{3 / 2} \exp \left[-\frac{\lambda}{1-\lambda \alpha_{\mathrm{W}}}\left(\boldsymbol{V}_{p \mathrm{q}}^{\mathrm{W}(0)}\right)^{2}\right] .
\end{aligned}
$$

Here $I_{\exp }^{(0)}\left(\boldsymbol{R}, \boldsymbol{R}^{\prime}\right)$ and $G_{\mathrm{W}}^{\exp (0)}(\boldsymbol{R}, \boldsymbol{P})$ are obtained from eqs. (A.4)-(A.9) by a simple change $f \rightarrow f^{(0)}, g \rightarrow g^{(0)}, h \rightarrow h^{(0)}$, leaving $h$ in $s(h)$ in its original form. Except for this particular phase $s(h)$, the superscript $(0)$ is always used to denote quantities evaluated with $\lambda=0$ in this appendix. For example, eqs. (A.17) and (A.18) yield

$$
\boldsymbol{V}_{p q}^{\mathbf{W}(0)}=\left[F_{p q}^{\mathbf{W}(0)}\left(\mu_{1} \mu_{0}\right)^{1 / 4}\left(\frac{\boldsymbol{R}}{b}\right)+G_{p q}^{\mathrm{W}(0)}\left(\mu_{1} \mu_{0}\right)^{-1 / 4} i\left(\frac{b \boldsymbol{P}}{\hbar}\right)\right] / D_{\mathrm{W}}^{(0)},
$$

with

$$
F_{p q}^{\mathrm{W}(0)}=\left(\frac{\mu_{0}}{\mu_{1}}\right)^{1 / 4}\left[\left(1+g^{(0)}\right) p+h^{(0)} q\right]+s(h)\left(\frac{\mu_{1}}{\mu_{0}}\right)^{1 / 4}\left[\left(1+f^{(0)}\right) q+h^{(0)} p\right],
$$

and so on. The new parameters $\alpha\left(\alpha_{\mathrm{w}}\right)$ in eq. (A.21) are given by

$$
\alpha=A_{p q}^{(0)} / D^{(0)}, \quad \alpha_{\mathrm{W}}=A_{p q}^{\mathrm{W}(0)} / D_{\mathrm{W}}^{(0)},
$$


with

$$
\begin{aligned}
A_{a b}^{(0)}= & F_{p q}^{(0)} a+G_{p q}^{(0)} b=F_{a b}^{(0)} p+G_{a b}^{(0)} q, \\
A_{a b}^{\mathrm{w}(0)}= & {\left[\left(\bar{\mu} g^{(0)}-\tilde{\mu}\right) p+\left(\bar{\mu} h^{(0)}+s(h)\right) q\right] a } \\
& +\left[\left(\bar{\mu} h^{(0)}+s(h)\right) p+\left(\bar{\mu} f^{(0)}+\tilde{\mu}\right) q\right] b .
\end{aligned}
$$

From eq. (A.21) one can rewrite all the coefficients in eqs. (A.5)-(A.8) in such a way that the $\lambda$-dependence is explicitly shown; for instance,

$$
\begin{aligned}
D_{\mathrm{W}} & =D_{\mathrm{w}}^{(0)}\left(1-\lambda \alpha_{\mathrm{w}}\right), \\
\eta & =\eta^{(0)}+\frac{\lambda}{1-\lambda \alpha_{\mathrm{w}}}\left(\mu_{1} \mu_{0}\right)^{1 / 2}\left(F_{p q}^{\mathrm{w}(0)} / D_{\mathrm{W}}^{(0)}\right)^{2}, \\
\zeta & =\zeta^{(0)}-\frac{\lambda}{1-\lambda \alpha_{\mathrm{W}}}\left(\mu_{1} \mu_{0}\right)^{-1 / 2}\left(G_{p q}^{\mathrm{w}(0)} / D_{\mathrm{w}}^{(0)}\right)^{2}, \\
\xi & =\xi^{(0)}-\frac{\lambda}{1-\lambda \alpha_{\mathrm{w}}} 2\left(F_{p q}^{\mathrm{w}(0)} / D_{\mathrm{w}}^{(0)}\right)\left(G_{p q}^{\mathrm{w}(0)} / D_{\mathrm{w}}^{(0)}\right) .
\end{aligned}
$$

One can also separate explicitly the $\lambda$-dependence contained in the polynomial terms by

$$
\begin{gathered}
a=a^{(0)}-\lambda p m, \quad b=b^{(0)}-\lambda q m, \quad c=c^{(0)}-\lambda p k, \\
d=d^{(0)}-\lambda q k, \quad e=e^{(0)}-\lambda m k .
\end{gathered}
$$

Then, $E\left(E_{\mathrm{W}}\right)$ and $\boldsymbol{V}_{a b}\left(\boldsymbol{V}_{a b}^{\mathrm{w}}\right)$ in eqs. (A.15)-(A.18) are expressed as

$$
\begin{aligned}
& E=E^{(0)}-\frac{\lambda}{1-\lambda \alpha}\left(A_{a^{(0)} b^{(0)} / D^{(0)}}^{(0)}-m\right)\left(A_{c^{(0)} d^{(0)} /}^{(0)} D^{(0)}-k\right), \\
& \boldsymbol{V}_{a b}=\boldsymbol{V}_{a^{(0)} b^{(0)}}^{(0)}+\frac{\lambda}{1-\lambda \alpha} \boldsymbol{V}_{p q}^{(0)}\left(A_{a^{(0)} b^{(0)}}^{(0)} / D^{(0)}-m\right) ; \\
& E_{\mathrm{W}}=E_{\mathrm{W}}^{(0)}-\frac{\lambda}{1-\lambda \alpha_{\mathrm{W}}}\left(A_{a^{(0)} b^{(0)}}^{\mathrm{w}(0)} D_{\mathrm{W}}^{(0)}-m\right)\left(A_{c^{(0)} d^{(0)}}^{\mathrm{w}(0)} / D_{\mathrm{W}}^{(0)}-k\right), \\
& \boldsymbol{V}_{a b}^{\mathrm{W}}=\boldsymbol{V}_{a^{(0)} b^{(0)}}^{\mathrm{W}(0)}+\frac{\lambda}{1-\lambda \alpha_{\mathrm{W}}} \boldsymbol{V}_{p q}^{\mathrm{W}(0)}\left(A_{a^{(0)} b^{(0)}}^{\mathrm{W}(0)} / D_{\mathrm{W}}^{(0)}-m\right) .
\end{aligned}
$$

The vectors $\boldsymbol{V}_{c d}$ and $\boldsymbol{V}_{\mathrm{cd}}^{\mathrm{W}}$ are obtained by a trivial change $a \rightarrow c, b \rightarrow d, m \rightarrow k$ in $\boldsymbol{V}_{a b}$ and $\boldsymbol{V}_{a b}^{\mathrm{W}}$, respectively. The coefficients for the $L S$ force are most compactly expressed by

$$
V^{L S}=\frac{4}{D^{(0)}} \frac{a d-b c}{1-\lambda \alpha}, \quad V_{\mathrm{W}}^{L S}=-s(h)\left(4 / D_{\mathrm{W}}^{(0)}\right) \frac{a d-b c}{1-\lambda \alpha_{\mathrm{W}}} .
$$


TABLE 4

The $L S$ Coefficients $\left(v^{L S} / \sqrt{5}\right)$

\begin{tabular}{|c|c|c|c|c|c|}
\hline No. ${ }^{a}$ ) & $0=D$ & $0=\mathbf{M}$ & No. & $\sigma=\mathrm{D}$ & $\mathcal{O}=\mathrm{M}$ \\
\hline \multirow{2}{*}{4} & $24 \kappa$ & $48(2+\kappa)$ & \multirow{2}{*}{16} & $24 \kappa$ & $12(1+\kappa)$ \\
\hline & $\overline{130+3 \kappa}$ & $\overline{130+3 \kappa}$ & & $\overline{21-\kappa}$ & $\overline{21-\kappa}$ \\
\hline \multirow{2}{*}{5} & $96 \kappa$ & $6(1-6 \kappa)$ & \multirow{2}{*}{17} & $72 \kappa$ & $36(1+\kappa)$ \\
\hline & $\overline{13(5+2 \kappa)}$ & $\overline{13(5+2 \kappa)}$ & & $\overline{7(12+5 k)}$ & $\overline{7(12+5 \kappa)}$ \\
\hline \multirow{2}{*}{6} & $48 \kappa$ & $6(5-8 \kappa)$ & \multirow{2}{*}{18} & $60 \kappa$ & $6(2-5 \kappa)$ \\
\hline & $\overline{65-6 \kappa}$ & $65-6 k$ & & $\overline{21+20 \kappa}$ & $-\overline{21+20 \kappa}$ \\
\hline \multirow{2}{*}{7} & $144 \kappa$ & $8(3-13 \kappa)$ & \multirow{2}{*}{19} & $96 \kappa$ & $8(3-8 \kappa)$ \\
\hline & $\overline{84-37 \kappa}$ & $\overline{84-37 \kappa}$ & & $\overline{84-37 \kappa}$ & $\overline{84-37 \kappa}$ \\
\hline \multirow{2}{*}{8} & $24 \kappa$ & $12(1+\kappa)$ & \multirow{2}{*}{20} & $6 \kappa$ & $3(1+\kappa)$ \\
\hline & $\overline{21-\kappa}$ & $\overline{21-\kappa}$ & & $\overline{21-\kappa}$ & $21-\kappa$ \\
\hline \multirow{2}{*}{9} & $48 \kappa$ & $24(1+\kappa)$ & $(21)$ & $48 \kappa$ & $24(1+\kappa)$ \\
\hline & $\overline{7(12+5 k)}$ & $\overline{7(12+5 \kappa)}$ & $(22)$ & $\overline{7(12+5 \kappa)}$ & $\overline{7(12+5 \kappa)}$ \\
\hline \multirow{2}{*}{10} & & $4(3-10 \kappa)$ & \multirow{2}{*}{23} & $30 \kappa$ & $3-5 \kappa$ \\
\hline & 0 & $21+20 \kappa$ & & $\overline{21+20 \kappa}$ & $\overline{21+20 \kappa}$ \\
\hline \multirow{2}{*}{11} & $56 \kappa$ & $4(4+17 \kappa)$ & \multirow{2}{*}{24} & $2 \kappa$ & $2(8+7 \kappa)$ \\
\hline & $\overline{130+3 \kappa}$ & $130+3 \kappa$ & & $\overrightarrow{13+4 \kappa}$ & $\overline{13+4 \kappa}$ \\
\hline \multirow{2}{*}{12} & $16 \kappa$ & $2(7-12 k)$ & \multirow{2}{*}{25} & $128 \kappa$ & $4(1+12 \kappa)$ \\
\hline & $13(5+2 \kappa)$ & $13(5+2 \kappa)$ & & $\overline{52+27 \kappa}$ & $\overline{52+27 \kappa}$ \\
\hline \multirow{2}{*}{13} & $32 \kappa$ & $2(5-9 k)$ & \multirow{2}{*}{26} & $128 \kappa$ & $4(1-3 \kappa)$ \\
\hline & $\overline{65-6 k}$ & $\overline{65-6 k}$ & & $\overline{52+27 \kappa}$ & $\overline{52+27 \kappa}$ \\
\hline \multirow{2}{*}{14} & $32 \kappa$ & $2(5+6 \kappa)$ & \multirow{2}{*}{27} & $32 \kappa$ & $4(11-18 \kappa)$ \\
\hline & $\overline{65-6 \kappa}$ & $\overline{65-6 \kappa}$ & & $\overline{52+27 \kappa}$ & $52+27 \kappa$ \\
\hline \multirow{2}{*}{15} & $264 \kappa$ & $4(9-19 \kappa)$ & \multirow{2}{*}{28} & $22 \kappa$ & $4-19 \kappa$ \\
\hline & $84-37 \kappa$ & $84-37 \kappa$ & & $\overline{13+4 \kappa}$ & $\overline{13+4 \kappa}$ \\
\hline
\end{tabular}

a) No. gives the exchange type (see table 1). Terms 1 through 3 have $v^{L S}=0$ both for $\theta=\mathrm{D}$ and $\mathrm{M}$.

TABLE 5

The D-type coefficients for the polynomial factors

\begin{tabular}{ccccc}
\hline No. & $v^{(0)} / \sqrt{5}$ & $v^{(2)} / \sqrt{5}$ & $u^{(c)} / \sqrt{5}$ & $u^{(s)} / \sqrt{5}$ \\
\hline 1 & $-\frac{12 \kappa}{3+\kappa}$ & 0 & $\frac{8 \kappa(3-4 \kappa)}{15(3+\kappa)^{2}}$ & 0 \\
2 & $-\frac{4 \kappa}{3+\kappa}$ & 0 & $\frac{4 \kappa(1-3 \kappa)}{15(3+\kappa)^{2}}$ & 0 \\
3 & $\frac{2 \kappa}{3+\kappa}$ & 0 & $\frac{4 \kappa(1+2 \kappa)}{15(31 \kappa)^{2}}$ & 0 \\
4 & $-\frac{240 \kappa}{130+3 \kappa}$ & $\frac{2400 \kappa(5+3 \kappa)}{(130+3 \kappa)^{2}}$ & $-\frac{16 \kappa(10+3 \kappa)}{(130+3 \kappa)^{2}}$ & $-\frac{8 \kappa(370+147 \kappa)}{(130+3 \kappa)^{2}}$
\end{tabular}


TABLE 5-continued

\begin{tabular}{|c|c|c|c|c|}
\hline No. & $v^{(0)} / \sqrt{5}$ & $v^{(2)} / \sqrt{5}$ & $\boldsymbol{u}^{(c)} / \sqrt{5}$ & $u^{(s)} / \sqrt{5}$ \\
\hline \multirow{2}{*}{5} & \multirow{2}{*}{0} & \multirow{2}{*}{0} & $16 \kappa$ & $32 \kappa$ \\
\hline & & & $39(5+2 \kappa)$ & $13(5+2 \kappa)$ \\
\hline \multirow{2}{*}{6} & $100 \kappa$ & $800 \kappa(15-11 \kappa)$ & $112 \kappa(15+4 \kappa)$ & $16 \kappa(165+254 \kappa)$ \\
\hline & $65-6 \kappa$ & $3(65-6 \kappa)^{2}$ & $27(65-6 \kappa)^{2}$ & $9(65-6 \kappa)^{2}$ \\
\hline \multirow{2}{*}{7} & $240 \kappa$ & $320 \kappa(12-91 \kappa)$ & $544 \kappa(24-47 \kappa)$ & $16 \kappa(1164-3427 \kappa)$ \\
\hline & $8 \overline{84-37 \kappa}$ & $\overline{(84-37 \kappa)^{2}}$ & $\overline{9(84-37 \kappa)^{2}}$ & $3(84-37 \kappa)^{2}$ \\
\hline \multirow{2}{*}{8} & $72 \kappa$ & $40 \kappa(3-13 \kappa)$ & $-16 \kappa(6+\kappa)$ & $8 \kappa(57+23 \kappa)$ \\
\hline & $\overline{21-\kappa}$ & $\overline{(21-\kappa)^{2}}$ & $\overline{9(21-\kappa)^{2}}$ & $3(21-\kappa)^{2}$ \\
\hline \multirow{2}{*}{9} & $48 \kappa$ & \multirow{2}{*}{0} & $32 \kappa(24-53 \kappa)$ & $16 \kappa$ \\
\hline & $\overline{12+5 \kappa}$ & & $\overline{21(12+5 \kappa)^{2}}$ & $\overline{7(12+5 \kappa)}$ \\
\hline \multirow{2}{*}{10} & $72 \kappa$ & $40 \kappa(3-10 \kappa)$ & $640 \kappa(3-10 \kappa)$ & $320 \kappa(3-10 \kappa)$ \\
\hline & $\overline{21+20 \kappa}$ & $\overline{(21+20 \kappa)^{2}}$ & $\overline{9(21+20 \kappa)^{2}}$ & $3(21+20 \kappa)^{2}$ \\
\hline \multirow{2}{*}{11} & $196 \kappa$ & $100 \kappa(20+57 \kappa)$ & $32 \kappa(17-3 \kappa)$ & $8 \kappa(790-321 \kappa)$ \\
\hline & $\overline{130+3 k}$ & $\overline{(130+3 \kappa)^{2}}$ & $\overline{3(130+3 \kappa)^{2}}$ & $\overline{3(130+3 \kappa)^{2}}$ \\
\hline \multirow{2}{*}{12} & $12 \kappa$ & \multirow[b]{2}{*}{0} & $16 \kappa(17-40 \kappa)$ & $16 \kappa$ \\
\hline & $\overline{5+2 \kappa}$ & & $\overline{585(5+2 \kappa)^{2}}$ & $39(5+2 \kappa)$ \\
\hline \multirow{2}{*}{13} & $138 \kappa$ & $400 \kappa(5-27 \kappa)$ & $112 \kappa(17-9 \kappa)$ & $32 \kappa(230-207 \kappa)$ \\
\hline & $\overline{65-6 k}$ & $3(65-6 k)^{2}$ & $\overline{27(65-6 \kappa)^{2}}$ & $9(65-6 k)^{2}$ \\
\hline \multirow{2}{*}{14} & $8 \kappa$ & $400 \kappa(5-2 \kappa)$ & $112(17-2 \kappa)$ & $64 \kappa(115-16 \kappa)$ \\
\hline & $\overline{65-6 \kappa}$ & $3(65-6 \kappa)^{2}$ & $\overline{27(65-6 \kappa)^{2}}$ & $9(65-6 \kappa)^{2}$ \\
\hline \multirow{2}{*}{15} & $116 \kappa$ & $160 \kappa(36+67 \kappa)$ & $272 \kappa(54-59 \kappa)$ & $8 \kappa(1548-3499 \kappa)$ \\
\hline & $\overline{84-37 \kappa}$ & $\overline{(84-37 \kappa)^{2}}$ & $\overline{9(84-37 \kappa)^{2}}$ & $3(84-37 \kappa)^{2}$ \\
\hline \multirow{2}{*}{16} & $10 \kappa$ & $20 \kappa(9-4 \kappa)$ & $-4 \kappa(27-2 \kappa)$ & $8 \kappa(72-7 \kappa)$ \\
\hline & $\overline{21-\kappa}$ & $\overline{(21-\kappa)^{2}}$ & $\overline{9(21-\kappa)^{2}}$ & $3(21-\kappa)^{2}$ \\
\hline \multirow[b]{2}{*}{17} & $20 \kappa$ & \multirow{2}{*}{0} & $32 \kappa(9-5 \kappa)$ & $24 \kappa$ \\
\hline & $\overline{12+5 \kappa}$ & & $\overline{7(12+5 \kappa)^{2}}$ & $\overline{7(12+5 \kappa)}$ \\
\hline \multirow{2}{*}{18} & $32 \kappa$ & $20 \kappa(9+20 \kappa)$ & $80 \kappa(27-20 \kappa)$ & $20 \kappa(9+100 \kappa)$ \\
\hline & $\overline{21+20 \kappa}$ & $\overline{(21+20 \kappa)^{2}}$ & $\overline{9(21+20 \kappa)^{2}}$ & $3(21+20 \kappa)^{2}$ \\
\hline \multirow{2}{*}{19} & $2 \kappa$ & $1920 \kappa(2 \kappa)$ & $272 \kappa(5-2 \kappa)$ & $32 \kappa(16-3 \kappa)$ \\
\hline & $\overline{84-37 \kappa}$ & $\overline{(84-37 \kappa)^{2}}$ & $\overline{3(84-37 \kappa)^{2}}$ & $\overline{(84-37 \kappa)^{2}}$ \\
\hline \multirow{2}{*}{20} & $23 \kappa$ & $10 \kappa(12-17 \kappa)$ & $2 \kappa(15-4 \kappa)$ & $2 \kappa(87-37 \kappa)$ \\
\hline & $\overline{21-\kappa}$ & $(21-\kappa)^{2}$ & $\overline{9(21-\kappa)^{2}}$ & $\overline{3(21-\kappa)^{2}}$ \\
\hline \multirow{2}{*}{21} & $14 \kappa$ & 0 & $16 \kappa(15+43 \kappa)$ & $16 \kappa$ \\
\hline & $\overline{12+5 \kappa}$ & 0 & $21(12+5 \kappa)^{2}$ & $\overline{7(12+5 \kappa)}$ \\
\hline \multirow{2}{*}{22} & $10 \kappa$ & 0 & $80 \kappa(3-4 \kappa)$ & $16 \kappa$ \\
\hline & $\overline{12+5 \kappa}$ & 0 & $\overline{21(12+5 \kappa)^{2}}$ & $\overline{7(12+5 \kappa)}$ \\
\hline \multirow{2}{*}{23} & $2 \kappa$ & $20 \kappa(6+5 \kappa)$ & $200 \kappa(3+4 \kappa)$ & $10 \kappa(33+20 \kappa)$ \\
\hline & $\overline{21+20 \kappa}$ & $\overline{(21+20 \kappa)^{2}}$ & $9(21+20 \kappa)^{2}$ & $3(21+20 \kappa)^{2}$ \\
\hline \multirow{2}{*}{24} & $14 \kappa$ & $5 \kappa(17+16 \kappa)$ & $224 \kappa(5+4 \kappa)$ & $2 \kappa(311+268 \kappa)$ \\
\hline & $\overline{13+4 \kappa}$ & $\overline{3(13+4 \kappa)^{2}}$ & $\overline{27(13+4 \kappa)^{2}}$ & $9(13+4 \kappa)^{2}$ \\
\hline 25 & $10 \kappa$ & $20 \kappa(53+28 \kappa)$ & $752 \kappa(1-4 \kappa)$ & $16 \kappa(1243+668 \kappa)$ \\
\hline & $\overline{52+27 \kappa}$ & $\overline{3(52+27 \kappa)^{2}}$ & $\overline{27(52+27 \kappa)^{2}}$ & $9(52+27 \kappa)^{2}$ \\
\hline
\end{tabular}


TABLE 5-continued

\begin{tabular}{ccccc}
\hline No. & $v^{(0)} / \sqrt{5}$ & $v^{(2)} / \sqrt{5}$ & $\mathfrak{u}^{(c)} / \sqrt{5}$ & $u^{(s)} / \sqrt{5}$ \\
\hline 26 & $-\frac{114 \kappa}{52+27 \kappa}$ & $\frac{20 \kappa(53+33 \kappa)}{3(52+27 \kappa)^{2}}$ & $\frac{752 \kappa(1-51 \kappa)}{27(52+27 \kappa)^{2}}$ & $-\frac{16 \kappa(1243+903 \kappa)}{9(52+27 \kappa)^{2}}$ \\
27 & $-\frac{142 \kappa}{52+27 \kappa}$ & $-\frac{20 \kappa(17+2 \kappa)}{3(52+27 \kappa)^{2}}$ & $\frac{752 \kappa(35-46 \kappa)}{27(52+27 \kappa)^{2}}$ & $\frac{16 \kappa(487-68 \kappa)}{9(52+27 \kappa)^{2}}$ \\
28 & $-\frac{16 \kappa}{13+4 \kappa}$ & $-\frac{5 \kappa(53+4 \kappa)}{3(13+4 \kappa)^{2}}$ & $-\frac{32 \kappa(1+20 \kappa)}{27(13+4 \kappa)^{2}}$ & $\frac{2 \kappa(419-68 \kappa)}{9(13+4 \kappa)^{2}}$ \\
\hline
\end{tabular}

TABLE 6

The $M$-type coefficients for the polynomial factors

\begin{tabular}{|c|c|c|c|c|}
\hline No. & $v^{(0)} / \sqrt{5}$ & $v^{(2)} / \sqrt{5}$ & $u^{(c)} / \sqrt{5}$ & $u^{(s)} / \sqrt{5}$ \\
\hline \multirow{2}{*}{1} & $4 \kappa$ & \multirow{2}{*}{0} & $4(1+\kappa)(3-4 \kappa)$ & \multirow{2}{*}{0} \\
\hline & $\overline{3+\kappa}$ & & $15(3+\kappa)^{2}$ & \\
\hline \multirow{2}{*}{2} & $1-\kappa$ & \multirow{2}{*}{0} & $2(1+\kappa)(1-3 \kappa)$ & \multirow{2}{*}{0} \\
\hline & $\overline{3+\kappa}$ & & $15(3+\kappa)^{2}$ & \\
\hline \multirow{2}{*}{3} & 2 & \multirow{2}{*}{0} & $2(1+\kappa)(1+2 \kappa)$ & \multirow{2}{*}{0} \\
\hline & $\overline{3+\kappa}$ & & $15(3+\kappa)^{2}$ & \\
\hline \multirow[b]{2}{*}{4} & $96 \kappa$ & $960 \kappa(5+3 \kappa)$ & $8(26+3 \kappa)(10+3 \kappa)$ & $16\left(260+236 \kappa+33 \kappa^{2}\right)$ \\
\hline & $\overline{130+3 \kappa}$ & $\overline{(130+3 \kappa)^{2}}$ & $3(130+3 \kappa)^{2}$ & $(130+3 \kappa)^{2}$ \\
\hline \multirow{2}{*}{5} & 12 & 60 & $4(3+22 \kappa)$ & $2(37+98 \kappa)$ \\
\hline & $\overline{13}$ & $\overline{169}$ & $\overline{507(5+2 \kappa)}$ & $\overline{169(5+2 \kappa)}$ \\
\hline \multirow[b]{2}{*}{6} & $4(5-12 \kappa)$ & $20(5+18 \kappa)(15-11 \kappa)$ & $4(55-18 \kappa)(15+4 \kappa)$ & $2\left(3675-2050 \kappa+1152 \kappa^{2}\right)$ \\
\hline & $65-6 \kappa$ & $3(65-6 \kappa)^{2}$ & $27(65-6 \kappa)^{2}$ & $9(65-6 \kappa)^{2}$ \\
\hline \multirow{2}{*}{7} & $130 \kappa$ & $520 \kappa(12-91 \kappa)$ & $16(63-83 \kappa)(24-47 \kappa)$ & $8\left(756-9849 \kappa+13663 \kappa^{2}\right)$ \\
\hline & $\overline{84-37 \kappa}$ & $\overline{3(84-37 \kappa)^{2}}$ & $27(84-37 \kappa)^{2}$ & $9(84-37 \kappa)^{2}$ \\
\hline \multirow[b]{2}{*}{8} & $2(3-19 \kappa)$ & $20(1+\kappa)(3-13 \kappa)$ & $8(1+\kappa)(6+\kappa)$ & $4(1+\kappa)(57+23 \kappa)$ \\
\hline & $21-\kappa$ & $(21-\kappa)^{2}$ & $9(21-\kappa)^{2}$ & $3(21-\kappa)^{2}$ \\
\hline \multirow{2}{*}{9} & $14 \kappa$ & \multirow{2}{*}{0} & $16(1+\kappa)(24-53 \kappa)$ & $8(1+\kappa)$ \\
\hline & $\overline{12+5 \kappa}$ & & $21(12+5 \kappa)^{2}$ & $\overline{7(12+5 \kappa)}$ \\
\hline \multirow[b]{2}{*}{10} & $2(3+14 \kappa)$ & $20(3+\kappa)(3-10 \kappa)$ & $16(3+40 \kappa)(3-10 \kappa)$ & $4(3-10 \kappa)(57-20 \kappa)$ \\
\hline & $21+20 \kappa$ & $3(21+20 \kappa)^{2}$ & $27(21+20 \kappa)^{2}$ & $9(21+20 \kappa)^{2}$ \\
\hline \multirow{2}{*}{11} & $26+79 \kappa$ & $40 \kappa(20+57 \kappa)$ & $16(26+3 \kappa)(17-3 \kappa)$ & $4\left(520+862 \kappa+291 \kappa^{2}\right)$ \\
\hline & $\overline{130+3 k}$ & $(130+3 \kappa)^{2}$ & $9(130+3 \kappa)^{2}$ & $3(130+3 \kappa)^{2}$ \\
\hline \multirow{2}{*}{12} & $40(2-7 \kappa)$ & \multirow[b]{2}{*}{0} & $4(3+22 \kappa)(17-40 \kappa)$ & $2(79-244 \kappa)$ \\
\hline & $65(5+2 \kappa)$ & & $1521(5+2 \kappa)^{2}$ & $-\overline{507(5+2 \kappa)}$ \\
\hline \multirow{2}{*}{13} & $25-66 \kappa$ & $10(5+18 \kappa)(5-27 \kappa)$ & $4(55-18 \kappa)(17-9 \kappa)$ & $2\left(125-4455 \kappa+1782 \kappa^{2}\right)$ \\
\hline & $\overline{65-6 \kappa}$ & $3(65-6 \kappa)^{2}$ & $27(65-6 \kappa)^{2}$ & $9(65-6 \kappa)^{2}$ \\
\hline \multirow{2}{*}{14} & 40 & $10(5+18 \kappa)(5-2 \kappa)$ & $4(55-18 \kappa)(17-2 \kappa)$ & $2\left(125-1880 \kappa+252 \kappa^{2}\right)$ \\
\hline & $\overline{65-6 \kappa}$ & $3(65-6 \kappa)^{2}$ & $27(65-6 \kappa)^{2}$ & $9(65-6 \kappa)^{2}$ \\
\hline \multirow{2}{*}{15} & $14-69 k$ & $260 \kappa(36+67 \kappa)$ & $8(63-83 \kappa)(54-59 \kappa)$ & $4\left(2268+8253 \kappa-13231 \kappa^{2}\right)$ \\
\hline & $84-37 \kappa$ & $3(84-37 \kappa)^{2}$ & $27(84-37 \kappa)^{2}$ & $9(84-37 \kappa)^{2}$ \\
\hline
\end{tabular}


TABLE 6-continued

\begin{tabular}{|c|c|c|c|c|}
\hline No. & $v^{(0)} / \sqrt{5}$ & $v^{(2)} / \sqrt{5}$ & $u^{(c)} / \sqrt{5}$ & $u^{(s)} / \sqrt{5}$ \\
\hline \multirow{2}{*}{16} & $2(8-3 \kappa)$ & $10(1+\kappa)(9-4 \kappa)$ & $2(1+\kappa)(27-2 \kappa)$ & $4(1+\kappa)(72-7 \kappa)$ \\
\hline & $\overline{21-\kappa}$ & $(21-\kappa)^{2}$ & $9(21-\kappa)^{2}$ & $\overline{3(21-\kappa)^{2}}$ \\
\hline \multirow{2}{*}{17} & $2-5 k$ & \multirow{2}{*}{0} & $16(1+\kappa)(9-5 \kappa)$ & $12(1+\kappa)$ \\
\hline & $\overline{12+5 \kappa}$ & & $7(12+5 \kappa)^{2}$ & $-\overline{7(12+5 \kappa)}$ \\
\hline \multirow{2}{*}{18} & $2(1-4 \kappa)$ & $10(3+\kappa)(9+20 \kappa)$ & $2(3+40 \kappa)(27-20 \kappa)$ & $2\left(432+255 \kappa+700 \kappa^{2}\right)$ \\
\hline & $\overline{21+20 \kappa}$ & $3(21+20 \kappa)^{2}$ & $27(21+20 \kappa)^{2}$ & $9(21+20 \kappa)^{2}$ \\
\hline \multirow{2}{*}{19} & $7-2 \kappa$ & $1040 \kappa(2-\kappa)$ & $8(63-83 \kappa)(5-2 \kappa)$ & $8\left(252-133 \kappa+36 \kappa^{2}\right)$ \\
\hline & $\overline{84-37 \kappa}$ & $\overline{(84-37 \kappa)^{2}}$ & $9(84-37 \kappa)^{2}$ & $3(84-37 \kappa)^{2}$ \\
\hline \multirow{2}{*}{20} & $-19-25 \kappa$ & $\underline{5(1+\kappa)(12-17 \kappa)}$ & $(1+\kappa)(15-4 \kappa)$ & $(1+\kappa)(87-37 \kappa)$ \\
\hline & $\overline{2(21-\kappa)}$ & $\overline{(21-\kappa)^{2}}$ & $9(21-\kappa)^{2}$ & $3(21-\kappa)^{2}$ \\
\hline \multirow{2}{*}{21} & $5-2 \kappa$ & \multirow{2}{*}{0} & $8(1+\kappa)(15+43 \kappa)$ & $8(1+\kappa)$ \\
\hline & $\overline{12+5 \kappa}$ & & $21(12+5 \kappa)^{2}$ & $\overline{7(12+5 \kappa)}$ \\
\hline \multirow{2}{*}{22} & 7 & \multirow{2}{*}{0} & $\underline{40(1+\kappa)(3-4 \kappa)}$ & $8(1+\kappa)$ \\
\hline & $\overline{12+5 k}$ & & $\overline{21(12+5 k)^{2}}$ & $\overline{7(12+5 \kappa)}$ \\
\hline \multirow{2}{*}{23} & $5+6 \kappa$ & $10(3+\kappa)(6+5 \kappa)$ & $5(3+40 \kappa)(3+4 \kappa)$ & $261+885 \kappa+500 \kappa^{2}$ \\
\hline & $\overline{2(21+20 \kappa)}$ & $3(21+20 \kappa)^{2}$ & $27(21+20 \kappa)^{2}$ & $9(21+20 \kappa)^{2}$ \\
\hline \multirow{2}{*}{24} & $13+32 \kappa$ & $5(13+8 \kappa)(17+16 \kappa)$ & $7(13-28 \kappa)(5+4 \kappa)$ & $1027+1744 \kappa+784 \kappa^{2}$ \\
\hline & $\overline{4(13+4 \kappa)}$ & $24(13+4 \kappa)^{2}$ & $54(13+4 \kappa)^{2}$ & $18(13+4 \kappa)^{2}$ \\
\hline \multirow{2}{*}{25} & $10(5+3 \kappa)$ & $20(5+3 \kappa)(53+28 \kappa)$ & $8(2+39 \kappa)(1-4 \kappa)$ & $4\left(56+2293 \kappa+1212 \kappa^{2}\right)$ \\
\hline & $\overline{52+27 \kappa}$ & $3(52+27 \kappa)^{2}$ & $27(52+27 \kappa)^{2}$ & $9(52+27 \kappa)^{2}$ \\
\hline \multirow{2}{*}{26} & $2-45 k$ & $20(5+3 \kappa)(53+33 \kappa)$ & $8(2+39 \kappa)(1-51 \kappa)$ & $4\left(56+4653 \kappa+2817 \kappa^{2}\right)$ \\
\hline & $\overline{52+27 \kappa}$ & $3(52+27 \kappa)^{2}$ & $27(52+27 \kappa)^{2}$ & $9(52+27 \kappa)^{2}$ \\
\hline \multirow{2}{*}{27} & $6(3-8 \kappa)$ & $20(5+3 \kappa)(17+2 \kappa)$ & $8(2+39 \kappa)(35-46 \kappa)$ & $4\left(1784-583 \kappa-1302 \kappa^{2}\right)$ \\
\hline & $52+27 \kappa$ & $3(52+27 \kappa)^{2}$ & $27(52+27 \kappa)^{2}$ & $9(52+27 \kappa)^{2}$ \\
\hline \multirow{2}{*}{28} & $13-28 \kappa$ & $5(13+8 \kappa)(53+4 \kappa)$ & $(13-28 \kappa)(1+20 \kappa)$ & $377-46 \kappa+344 \kappa^{2}$ \\
\hline & $\overline{4(13+4 \kappa)}$ & $24(13+4 \kappa)^{2}$ & $54(13+4 \kappa)^{2}$ & $18(13+4 \kappa)^{2}$ \\
\hline
\end{tabular}

The explicit separation of the $\lambda$-dependence in the exchange kernels has the advantage that it makes possible the evaluation of the exchange kernels for almost any kind of functional form for $u(r)$, as long as it can be expressed by gaussian integrals. Since the case $\lambda=0$ also corresponds to the normalization kernel, this method is the most economical way to calculate all necessary exchange kernels in complete analytic form.

\section{Appendix B}

The coefficients $v^{L S}, v^{(0)}, v^{(2)}, u^{(c)}, u^{(s)}$ needed for the Wigner transforms of the coupling kernels, eq. (16), are given as functions of $\kappa=\chi^{2} b^{2} /\left(1+2 \chi^{2} b^{2}\right)$ and exchange and interaction type through tables 4-6. 


\section{References}

1) Y. Fujiwara and K.T. Hecht, Nucl. Phys. A444 (1985) 541

2) N. Isgur, Nuclear physics from the quark model with chromodynamics, University of Toronto Preprint $85-18$, to be publ.;

S. Godfrey and N. Isgur, Phys. Rev. D32 (1985) 189

3) Y. Fujiwara and K.T. Hecht, Nucl. Phys. A451 (1986) 625

4) Y. Fujiwara and K.T. Hecht, Phys. Lett. 171B (1986) 17

5) Y. Suzuki and K.T. Hecht, Nucl. Phys. A420 (1984) 525; A446 (1985) 749

6) H. Horiuchi, Prog. Theor. Phys. 55 (1976) 1448.

7) T.H. Seligman and W. Zahn, J. of Phys. G2 (1976) 79

8) P. Kramer and T.H. Seligman, Nucl. Phys. A136 (1969) 545; A186 (1972) 49

9) Y. Fujiwara and Y.C. Tang, University of Minnesota report, UM-RGM2 (1984)

10) K. Aoki and H. Horiuchi, Prog. Theor. Phys. 68 (1982) 1658, 2028

11) Y. Suzuki, Nucl. Phys. A405 (1983) 40

12) J. Burger and H.M. Hofmann, Phys. Lett. 148B (1984) 25

13) Y. Yamauchi, R. Yamamoto and M. Wakamatsu, Nucl. Phys. A443 (1985) 628

14) H.J. Pirner, Phys. Lett 85B (1979) 190;

C.S. Warke and R. Shanker, Phys. Rev. C21 (1980) 2643;

O. Morimatsu, S. Ohta, K. Shimizu and K. Yazaki, Nucl. Phys. A420 (1984) 573;

O. Morimatsu, K. Yazaki and M. Oka, Nucl. Phys. A424 (1984) 412;

F. Wang and C.W. Wong, Nucl. Phys. A438 (1985) 620;

K. Holinde, Phys. Lett. 157B (1985) 123;

Y. He, F. Wang and C.W. Wong, Nucl. Phys. A451 (1986) 653 